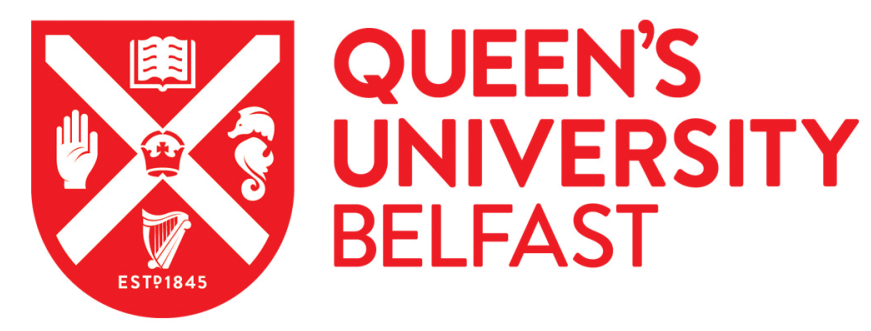

\title{
On Generalizing Collective Spatial Keyword Queries
}

Chan, H. K-H., Long, C., \& Wong, R. C-W. (2018). On Generalizing Collective Spatial Keyword Queries. IEEE Transactions on Knowledge and Data Engineering, 30(9), 1712-1726.

https://doi.org/10.1109/TKDE.2018.2800746

\section{Published in:}

IEEE Transactions on Knowledge and Data Engineering

\section{Document Version:}

Peer reviewed version

\section{Queen's University Belfast - Research Portal:}

Link to publication record in Queen's University Belfast Research Portal

\section{Publisher rights}

(c) 2018 IEEE.

This work is made available online in accordance with the publisher's policies. Please refer to any applicable terms of use of the publisher.

\section{General rights}

Copyright for the publications made accessible via the Queen's University Belfast Research Portal is retained by the author(s) and / or other copyright owners and it is a condition of accessing these publications that users recognise and abide by the legal requirements associated with these rights.

Take down policy

The Research Portal is Queen's institutional repository that provides access to Queen's research output. Every effort has been made to ensure that content in the Research Portal does not infringe any person's rights, or applicable UK laws. If you discover content in the Research Portal that you believe breaches copyright or violates any law, please contact openaccess@qub.ac.uk. 


\title{
On Generalizing Collective Spatial Keyword Queries
}

\author{
Harry Kai-Ho Chan, Cheng Long, and Raymond Chi-Wing Wong
}

\begin{abstract}
With the proliferation of spatial-textual data such as location-based services and geo-tagged websites, spatial keyword queries are ubiquitous in real life. One example of spatial-keyword query is the so-called collective spatial keyword query (CoSKQ) which is to find for a given query consisting a query location and several query keywords a set of objects which covers the query keywords collectively and has the smallest cost wrt the query location. In the literature, many different functions were proposed for defining the cost and correspondingly, many different approaches were developed for the CoSKQ problem. In this paper, we study the CoSKQ problem systematically by proposing a unified cost function and a unified approach for the CoSKQ problem (with the unified cost function). The unified cost function includes all existing cost functions as special cases and the unified approach solves the CoSKQ problem with the unified cost function in a unified way. Experiments were conducted on both real and synthetic datasets which verified our proposed approach.
\end{abstract}

Index Terms-Spatial keyword queries, unified framework

\section{INTRODUCTION}

$\mathrm{N}$ OWADAYS, geo-textual data which refers to data with both spatial and textual information is ubiquitous. Some examples of geo-textual data include the spatial points of interest (POI) with textual description (e.g., restaurants, cinema, tourist attractions, and hotels), geo-tagged web objects (e.g., webpages and photos at Flickr), and also geo-social networking data (e.g., users of FourSquare have their check-in histories which are spatial and also profiles which are textual).

One application based on geo-textual data is to search a set of (geo-textual) objects wrt a query consisting of a query location (e.g., the location one is located at) and some textual information (e.g., some keywords expressing the targets one wants to search) such that the objects have their textual information matching the query keywords and their locations close to the query location. One scenario of this application is that a tourist wants to find several POIs such that s/he could do sight-seeing, shopping and dining and the POIs are close to the hotel. In this case, the user can set the query location to the hotel location and the query keywords to be "attractions", "shopping" and "restaurant" to search for a set of POIs. Another scenario is that a manager wants to set up a project consortium of partners close to each other such that they together offer the capabilities required for successful execution of the whole project. In this case, the user can issue the query with his/her location as the query location and the required skills for the partners as the query keywords to find a group of people.

The above applications were captured by the so-called Collective Spatial Keyword Query (CoSKQ) [3], [17], [2] in the literature. Let $\mathcal{O}$ be a set of objects, where each object $o \in \mathcal{O}$ is associated with a spatial location, denoted by $0 . \lambda$, and a set of keywords, denoted by $o . \psi$. Given a query $q$ with a location $q . \lambda$

- H.K.-H. Chan and R.C.-W. Wong are with the Department of Computer Science and Engineering, The Hong Kong University of Science and Technology, Clear Water Bay, Kowloon, Hong Kong.

E-mail: $\{$ khchanak, raywong\}@ cse.ust.hk

- C. Long is with the School of Electronics, Electrical Engineering and Computer Science, Queen's University Belfast, BT7 1NN, Northern Ireland, United Kingdom. E-mail: cheng.long@qub.ac.uk and a set of keywords $q . \psi$, the CoSKQ problem is to find a set $S$ of objects such that $S$ covers $q . \psi$, i.e., $q . \psi \subseteq \cup_{o \in S} 0 . \psi$, and the cost of $S$, denoted by $\operatorname{cost}(S)$, is minimized.

In the literature, many different cost functions have been proposed for $\operatorname{cost}(S)$ in the CoSKQ problem, and these cost functions are applicable in different scenarios in addition to the above examples. For the CoSKQ problem with each particular cost function, at least one approach has been designed, which we briefly review as follows.

Different cost functions. Five different cost functions have been proposed for the CoSKQ problem, namely, cost $_{S u m}$ [3],

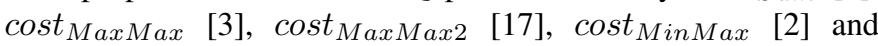
cost $_{\text {SumMax }}$ [2]. For example, $\operatorname{cost}_{\text {Sum }}(S)$ defines the cost to the summation of the distances from the query location to the objects in $S$, and $\operatorname{cost}_{\text {MaxMax }}(S)$ defines the cost to a linear combination of the maximum distance between the query location and an object in $S$ and the maximum pairwise distance among the objects in $S$. The definitions of the rest of cost functions would be introduced later. Each cost function has its own semantic meaning and depending on the application scenario, an appropriate cost function is used.

Different approaches. For the CoSKQ problem with each of these existing cost functions, which was proved to be NP-hard, at least one solution (including an exact algorithm and an approximate algorithm) was developed, and these solutions usually differ from one another. For example, the exact algorithm for the CoSKQ problem with cost $_{S u m}$ is a dynamic programming algorithm [3], while that for the one with cost $_{\text {MaxMax }}$ is a branch-and-bound algorithm [3]. Usually, an existing algorithm for the CoSKQ problem with a particular cost function cannot be used to solve that with another cost function.

In this paper, we study the CoSKQ problem systematically by proposing a unified cost function and a unified approach for the CoSKQ problem (with the unified cost function).

Without the unified approach, we need to handle different cost functions by different algorithms, which increases the difficulty 
for CoSKQ to be used in practice. Also, when researchers work on improving the performance of an algorithm, only the corresponding cost function is benefited. Although sometimes it is possible that one algorithm originally designed for one cost function can be adapted for another cost function, the performance of the adapted algorithm is not satisfactory. A better idea is to have a unified cost function and a unified approach, where the unified cost function captures all known cost functions and some other cost functions which are not known before but useful.

Specifically, the main contribution is summarized as follows.

A unified cost function. We propose a unified cost function cost $_{\text {unified }}$ which expresses all existing cost functions and a few new cost functions that have not been studied before. The core idea

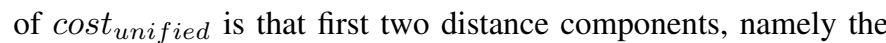
query-object distance component and the object-object distance component, are defined, where the former is based on the distances between the query location and those of the objects and the latter is based on the pairwise distances among the set of objects and then cost $_{\text {unified }}$ is defined based on the two distance components carefully such that all existing cost functions are captured (Note that this is possible since all ingredients of defining a cost function are distances between the query location and and those distances among objects which are captured by the two components.).

A unified approach. We design a unified approach, which consists of one exact algorithm and one approximate algorithm, for the CoSKQ problem with the unified cost function. For the CoSKQ problem with the cost function instantiated to those existing cost functions, which have been proved to be NP-hard, our exact algorithm is superior over the state-of-the-arts in that it not only has a unified procedure, but also runs faster under all settings for some cost functions (e.g., $\operatorname{cost}_{\text {MinMax }}$ and $\operatorname{cost}_{\operatorname{MinMax2}}$ ) and under the majority of settings for the other cost functions, and our approximate algorithm is always among those algorithms which give the best approximation ratios and runs faster than those algorithms which give similar approximation ratios. For the CoSKQ problem with the cost function instantiated to those new cost functions that have not been studied before, our exact algorithm runs reasonably fast and our approximate algorithm provides certain approximation ratios.

Besides, we conducted extensive experiments based on both real and synthetic datasets which verified our unified approach.

The rest of this paper is organized as follows. Section 2 gives the related work. Section 3 introduces the unified cost function and Section 4 presents the unified approach for CoSKQ. Section 5 gives the empirical study and Section 6 concludes the paper.

\section{Related Work}

Many existing studies on spatial keyword queries focus on retrieving a single object that is close to the query location and relevant to the query keywords.

A boolean kNN query [12], [5], [24], [30], [27] finds a list of $k$ objects each covering all specified query keywords. The objects in the list are ranked based on their spatial proximity to the query location.

A top- $k$ kNN query [8], [18], [15], [19], [20], [9], [25] adopts the ranking function considering both the spatial proximity and the textual relevance of the objects and returns top- $k$ objects based on the ranking function. This type of queries has been studied on Euclidean space [8], [18], [15], road network databases [19], trajectory databases [20], [9] and moving object databases [25]. Usually, the methods for this kind of queries adopt an index structure called the IR-tree [8], [23] capturing both the spatial proximity and the textual information of the objects to speed up the keyword-based nearest neighbor $(\mathrm{NN})$ queries and range queries. In this paper, we also adopt the IR-tree for keyword-based NN queries and range queries.

Some other studies on spatial keyword queries focus on finding an object set as a solution. Among them, some [3], [17], [2] studied the collective spatial keyword queries (CoSKQ). Cao et al. [3], [2] proposed four cost functions, namely cost Sum $_{\text {, }}$ cost $_{\text {MaxMax }}, \operatorname{cost}_{\text {MinMax }}$ and $\operatorname{cost}_{\text {SumMax }}$, and developed algorithms for the CoSKQ problem with the first three cost functions, leaving that with the fourth cost function, i.e., cost $_{\text {SumMax }}$, as future work. Besides, they studied two variations of CoSKQ, namely top- $k$ CoSKQ and weighted CoSKQ, in [2]. Long et al. [17] proposed exact and approximate algorithms for the CoSKQ problem with cost MaxMax $_{\text {Max }}$ and also that with a new cost function $\operatorname{cost}_{\operatorname{MaxMax2} 2}$. The details of these cost functions are described in Section 3 In this paper, we also study the CoSKQ problem. Specifically, we propose a unified cost function which include all existing cost functions as special cases and based on the unified cost function, we design a unified approach, consisting of an exact algorithm and an approximate algorithm.

Another query that is similar to the CoSKQ problem is the $m C K$ query [28], [29], [14] which takes a set of $m$ keywords as input and finds $m$ objects with the minimum diameter that cover the $m$ keywords specified in the query. In the existing studies of $m \mathrm{CK}$ queries, it is usually assumed that each object contains a single keyword. There are some variants of the $m \mathrm{CK}$ query, including the SK-COVER [7] and the BKC query [10]. These queries are similar to the CoSKQ problem in that they also return an object set that covers the query keywords, but they only take a set of keywords as input. In contrast, the CoSKQ problem studied in this paper takes both a set of keywords and a spatial location as inputs.

Skovsgaard et al. [21] proposed a query to find top- $k$ groups of objects with the ranking function considering the spatial proximity and textual relevance of the groups. Liu et al. proposed the cluebased spatio-textual query [16] which takes a set of keywords and a clue as inputs, and returns $k$ objects with highest similarities against the clue.

There are also some studies [13], [22] on spatial keyword queries which find an object set in the road network, some [6] which find an object set with the scoring function considering an inherent cost in each object, some [4], [11] which find a region as a solution and some [1], [26] which find a route as a solution.

\section{A Unified Cost Function}

Let $\mathcal{O}$ be a set of objects, where each object $o \in \mathcal{O}$ is associated with a spatial location, denoted by $o . \lambda$, and a set of keywords, denoted by $o . \psi$. Given two objects $o_{1}$ and $o_{2}$, we denote by $d\left(o_{1}, o_{2}\right)$ the Euclidean distance between $o_{1} \cdot \lambda$ and $o_{2} \cdot \lambda$.

(1) Problem definition. A collective spatial keyword query (CoSKQ) [3] is defined as follows.

Problem 1 (CoSKQ [3]). Given a query $q$ with a location $q . \lambda$ and a set of keywords $q . \psi$, the CoSKQ problem is to find a set $S$ of objects such that $S$ covers $q . \psi$, i.e., $q . \psi \subseteq \cup_{o \in S} o . \psi$, and the cost of $S$, denoted by $\operatorname{cost}(S)$, is minimized.

(2) Existing cost functions. To the best of our knowledge, five cost functions have been proposed for defining $\operatorname{cost}(\cdot)$ in 


\begin{tabular}{|c|c|c|c|c|c|}
\hline & \multicolumn{3}{|c|}{ Parameter } & \multirow{2}{*}{$\operatorname{cost}_{\text {unified }}\left(S \mid \alpha, \phi_{1}, \phi_{2}\right)$} & \multirow{2}{*}{ Existing/New } \\
\hline & $\alpha \in(0,1]$ & $\phi_{1} \in\{1, \infty,-\infty\}$ & $\phi_{2} \in\{1, \infty\}$ & & \\
\hline $\mathrm{a}$ & $0.5^{*}$ & 1 & 1 & $\sum_{o \in S} d(o, q)+\max _{o_{1}, o_{2} \in S} d\left(o_{1}, o_{2}\right)$ & cost $_{\text {SumMax }}$ |2] \\
\hline $\mathrm{b}$ & $0.5^{*}$ & 1 & $\infty$ & $\max \left\{\sum_{o \in S} d(o, q), \max _{o_{1}, o_{2} \in S} d\left(o_{1}, o_{2}\right)\right\}$ & cost $_{\text {SumMax2 }}$ (New) \\
\hline $\mathrm{c}$ & $0.5^{*}$ & $\infty$ & 1 & $\max _{o \in S} d(o, q)+\max _{o_{1}, o_{2} \in S} d\left(o_{1}, o_{2}\right)$ & $\operatorname{cost}_{\operatorname{Max} M a x}$ [3], [17], [2] \\
\hline $\mathrm{d}$ & $0.5^{*}$ & $\infty$ & $\infty$ & $\max \left\{\max _{o \in S} d(o, q), \max _{o_{1}, o_{2} \in S} d\left(o_{1}, o_{2}\right)\right\}$ & $\operatorname{cost}_{\operatorname{Max} \operatorname{Max2} 2}$ \\
\hline $\mathrm{e}$ & $0.5^{*}$ & $-\infty$ & 1 & $\min _{o \in S} d(o, q)+\max _{o_{1}, o_{2} \in S} d\left(o_{1}, o_{2}\right)$ & cost $_{\text {MinMax }}$ [2] \\
\hline $\mathrm{h}$ & 1 & $\infty$ & - & $\max _{o \in S} d(o, q)$ & $\operatorname{cost}_{\operatorname{Max}}(\mathrm{New})$ \\
\hline $\mathrm{i}$ & 1 & $-\infty$ & - & $\min _{o \in S} d(o, q)$ & $\operatorname{cost}_{M i n}(\mathrm{New})$ \\
\hline
\end{tabular}

Following the existing studies, $\alpha=0.5$ is used to illustrate the case of $\alpha \in(0,1)$ for simplicity

TABLE 1: cost $_{\text {unified }}$ under different parameter settings

the CoSKQ problem, namely cost $_{\text {Sum }}$ [3], cost SumMax $_{\text {[2], }}$

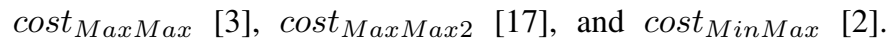
Specifically, these cost functions are defined as follows.

1) $\operatorname{cost}_{S u m} \cdot \operatorname{cost}_{\text {Sum }}(S)$ defines the cost to be the summation of the distances from the query location to the objects in $S$, i.e., $\operatorname{cost}_{S u m}(S)=\sum_{o \in S} d(o, q)$.

2) $\operatorname{cost}_{\text {SumMax }} \cdot \operatorname{cost}_{\text {SumMax }}(S)$ defines the cost to be a linear combination of the summation of distances from the query location to the objects in $S$ and the maximum pairwise distance among the objects in $S$, i.e., $\operatorname{cost}_{\text {SumMax }}(S)=\alpha \cdot \sum_{o \in S} d(o, q)+(1-\alpha)$. $\max _{O_{1}, o_{2} \in S} d\left(o_{1}, o_{2}\right)$, where $\alpha$ represents a real number in $(0,1]$.

3) $\operatorname{cost}_{\text {MaxMax }} \cdot \operatorname{cost}_{\operatorname{Max} M a x}(S)$ defines the cost to be a linear combination of the maximum distance between the query location and an object in $S$ and the maximum pairwise distance among the objects in $S$, i.e., $\operatorname{cost}_{\text {MaxMax }}(S)=\alpha \cdot \max _{o \in S} d(o, q)+(1-\alpha)$. $\max _{o_{1}, o_{2} \in S} d\left(o_{1}, o_{2}\right)$, where $\alpha$ represents a real number in $(0,1]$.

4) $\operatorname{cost}_{\operatorname{MaxMax2}} \cdot \operatorname{cost}_{\operatorname{Max} \operatorname{Max} 2}(S)$ defines the cost to be the larger one of the maximum distance between the query location and an object in $S$ and the maximum pairwise distance among the objects in $S$, i.e., $\operatorname{cost}_{\operatorname{MaxMax} 2}(S)=$ $\max \left\{\max _{o \in S} d(o, q), \max _{o_{1}, o_{2} \in S} d\left(o_{1}, o_{2}\right)\right\}$.

5) $\operatorname{cost}_{\text {MinMax }} \cdot \operatorname{cost}_{\text {MinMax }}(S)$ defines the cost to be a linear combination of the minimum distance between the query location and an object in $S$ and the maximum pairwise distance among the objects in $S$, i.e., $\operatorname{cost}_{\text {MinMax }}(S)=\alpha \cdot \min _{o \in S} d(o, q)+(1-\alpha)$. $\max _{o_{1}, o_{2} \in S} d\left(o_{1}, o_{2}\right)$, where $\alpha$ represents a real number in $(0,1]$.

(3) A unified cost function cost $_{\text {unified }}$. In this paper, we propose a unified cost function cost $t_{\text {unified }}$ which could be instantiated to many different cost functions including all those five existing ones. Before we give the exact definition of cost $_{\text {unified }}$, we first introduce a distance component used for defining cost $_{\text {unified, }}$, namely the query-object distance component. It is defined based on the distances between the query location and the objects in $S$. Specifically, we denote it by $D_{q, o}\left(S \mid \phi_{1}\right)$ and define it as follows.

$$
D_{q, o}\left(S \mid \phi_{1}\right)=\left[\sum_{o \in S}(d(o, q))^{\phi_{1}}\right]^{\frac{1}{\phi_{1}}}
$$

where $\phi_{1} \in\{1, \infty,-\infty\}$ is a user parameter. Depending on the setting of $\phi_{1}, D_{q, o}\left(S \mid \phi_{1}\right)$ corresponds to the summation, the max- imum, or the minimum of the distances from the query location to the objects in $S$. Specifically,

$$
D_{q, o}\left(S \mid \phi_{1}\right)= \begin{cases}\sum_{o \in S} d(o, q), & \text { if } \phi_{1}=1 \\ \max _{o \in S} d(o, q), & \text { if } \phi_{1}=\infty \\ \min _{o \in S} d(o, q), & \text { if } \phi_{1}=-\infty\end{cases}
$$

With the distance component defined, we are ready to introduce the unified cost function cost $_{\text {unified. }}$. Specifically, we define cost $_{\text {unified }}$ as follows.

$$
\begin{aligned}
& \text { cost }_{\text {unified }}\left(S \mid \alpha, \phi_{1}, \phi_{2}\right) \\
& =\left\{\left[\alpha \cdot D_{q, o}\left(S \mid \phi_{1}\right)\right]^{\phi_{2}}+\left[(1-\alpha) \max _{o_{1}, o_{2} \in S} d\left(o_{1}, o_{2}\right)\right]^{\phi_{2}}\right\}^{\frac{1}{\phi_{2}}}
\end{aligned}
$$

where $\alpha \in(0,1]^{1}, \phi_{1} \in\{1, \infty,-\infty\}$ and $\phi_{2} \in\{1, \infty\}$ are user parameters. In the following, we write $\operatorname{cost}_{\text {unified }}\left(S \mid \alpha, \phi_{1}, \phi_{2}\right)$ simply as $\operatorname{cost}(S)$ when there is no ambiguity.

Same as [3], [17], [2], for ease of exposition, we use $\alpha=0.5$ to illustrate the case of $\alpha \in(0,1)$. In this case, we can safely assume that

$$
\begin{aligned}
& \text { cost }_{\text {unified }}\left(S \mid 0.5, \phi_{1}, \phi_{2}\right) \\
& =\left\{\left[D_{q, o}\left(S \mid \phi_{1}\right)\right]^{\phi_{2}}+\left[\max _{o_{1}, o_{2} \in S} d\left(o_{1}, o_{2}\right)\right]^{\phi_{2}}\right\}^{\frac{1}{\phi_{2}}}
\end{aligned}
$$

Under some settings of $\alpha, \phi_{1}$ and $\phi_{2}$, cost unified $_{\text {corresponds }}$ to one of the aforementioned existing cost functions (as shown in Table 1). For example, when $\alpha=1$ and $\phi_{1}=1$ (regardless of the settings of $\left.\phi_{2}\right)$, cost $t_{\text {unified }}(S)$ corresponds to $\operatorname{cost}_{S u m}(S)$ since

$$
\begin{aligned}
\operatorname{cost}_{\text {unified }}(S) & =\left\{\left[D_{q, o}(S \mid 1)\right]^{\phi_{2}}\right\}^{\frac{1}{\phi_{2}}}=D_{q, o}(S \mid 1) \\
& =\sum_{o \in S} d(o, q)=\operatorname{cost}_{S u m}(S)
\end{aligned}
$$

and similarly, when $\alpha \in(0,1], \phi_{1}=\infty$ and $\phi_{2}=1$, $\operatorname{cost}_{\text {unified }}(S)$ corresponds to $\operatorname{cost}_{\text {MaxMax }}(S)$.

Under some other settings of $\alpha, \phi_{1}$ and $\phi_{2}$, cost $_{\text {unified }}$ corresponds to a new cost function that has not been studied before. For example, when $\alpha=0.5, \phi_{1}=1$, and $\phi_{2}=\infty$, we have

$$
\begin{aligned}
\operatorname{cost}_{\text {unified }}(S) & =\left\{\left[0.5 \cdot D_{q, o}(S \mid 1)\right]^{\infty}+\left[0.5 \cdot \max _{o_{1}, o_{2} \in S} d\left(o_{1}, o_{2}\right)\right]^{\infty}\right\}^{\frac{1}{\infty}} \\
& =0.5 \max \left\{\sum_{o \in S} d(o, q), \max _{o_{1}, o_{2} \in S} d\left(o_{1}, o_{2}\right)\right\}
\end{aligned}
$$

where we denote $\max \left\{\sum_{o \in S} d(o, q), \max _{o_{1}, o_{2} \in S} d\left(o_{1}, o_{2}\right)\right\}$ by cost $_{\text {SumMax } 2}(S)$.

The instantiations of cost $_{\text {unified }}$ depending on different parameter settings are shown in Table 1 . In the following, we introduce those instantiations that are new.

1. In the setting of $\alpha=0$, the query location has no contribution to the cost Thus, we do not consider this setting. 
1) (row b) $\operatorname{cost}_{\operatorname{SumMax} 2}$. The functionality of this cost function is equivalent to that of the cost function $\operatorname{cost}_{\text {Sum }}$ (please see Appendix A for details), and thus we focus on cost $_{\text {Sum }}$ in this paper.

2) (row f) $\operatorname{cost}_{\operatorname{MinMax} 2}$. It essentially captures the maximum among two distances, namely the distance between the query location $q . \lambda$ and its nearest object in $S$ and the distance between the two farthest objects in $S$. A common practice for an individual to explore the objects returned is to visit the object which is the nearest from the query location and explore the others, and thus this cost function is useful when people want to get at their first stop (i.e., the nearest object) fastly (this is captured by the query-object distance component) and explore the objects within a small region (this is captured by the farthest pairwise distance of the objects). Compared to the existing cost function $\operatorname{cost}_{\text {MinMax }}$, cost $_{\text {MinMax } 2}$ has an advantage that it requires no parameter of $\alpha$.

3) (row h) $\operatorname{cost}_{\operatorname{Max}}$. It uses the maximum distance between the query location $q . \lambda$ and an object in $S$. This cost function can be used to find the feasible set with the closet farthest object among all feasible sets. This cost function is suitable for the scenarios where a user visits one object a time, starting from the query location each time, and wants the worst-case cost as small as possible.

4) (row i) $\operatorname{cost}_{\mathrm{Min}}$. It uses the distance between the query location $q . \lambda$ and its nearest object in $S$ only, which is of no interest in practice since it put no penalty on those objects that are far away from the query location, e.g., the whole set of objects corresponds to a trivial solution for the CoSKQ problem with cost $_{M i n}$. Therefore, we ignore this instantiation of cost $t_{\text {unified }}$.

(4) Intractability results. It is known that the CoSKQ problem with an existing cost function adopted is NP-hard [3], [17], [2]. That is, the CoSKQ problem is NP-hard under the parameter settings such that cost $_{\text {unified }}$ corresponds to an existing cost function. In this paper, we study the intractability of the CoSKQ problem with all possible parameter settings of $\alpha, \phi_{1}$ and $\phi_{2}$ for cost $_{\text {unified. }}$. Specifically, we have the following result.

Theorem 1 (Intractability). The CoSKQ problem is NP-hard with all possible parameter settings of $\alpha, \phi_{1}$ and $\phi_{2}$ except for the setting of $\alpha=1, \phi_{1} \in\{\infty,-\infty\}$.

Proof. See Appendix B

(5) Existing Algorithms. For the CoSKQ problem with each of the existing cost functions, solution (including an exact algorithm and an approximate algorithm) was developed, and these solutions usually differ from one another. Specifically, we review the algorithms of some existing cost functions and solutions as follows.

1) $\operatorname{cost}_{S u m}$. The exact algorithm for CoSKQ problem with cost $_{S u m}$ is a dynamic programming algorithm, while the approximate algorithm is a greedy algorithm transformed from that of the Weighted Set Cover problem [3], [2]

2) $\operatorname{cost}_{\text {SumMax }}$. No solution is available in the literature for solving CoSKQ with $\operatorname{cost}_{\text {SumMax }}$. This cost function is proposed in [2], but the corresponding solution is left for their future work.

3) $\operatorname{cost}_{\text {MaxMax }}$. Several algorithms were proposed for CoSKQ problem with $\operatorname{cost}_{\text {MaxMax }}$. One of the exact algorithms is a branch-and-bound algorithm [3], while another one is based on a distance owner-driven approach [17]. One of the approximate algorithms picks the nearest neighbor set [3], [2], while two other approximate algorithms search for feasible sets in an iterative manner [3], [17], [2].

Usually, an existing algorithm for the CoSKQ problem with a particular cost function cannot be used to solve that with another cost function. In the following section, we introduce out unified approach for the CoSKQ problem with the unified cost function.

\section{A Unified Approach}

In this section, we introduce our unified approach which consists of one exact algorithm called Unified-E (Section 4.1) and one approximate algorithm called Unified-A (Section 4.2). While the unified cost function combines existing ones, our unified approach is not one which simply combine existing approaches. In fact, both the exact algorithm and approximate algorithm proposed in this paper are clean and elegant while existing approaches have quite different structures.

Before presenting the algorithms, we first give some definitions as follows. Given a query $q$ and an object $o$ in $\mathcal{O}$, we say $o$ is a relevant object if $o . \psi \cap q . \psi \neq \emptyset$. We denote $\mathcal{O}_{q}$ to be the set of all relevant objects. Given a set $S$ of objects, $S$ is said to be a feasible set if $S$ covers $q . \psi$ (i.e. $q . \psi \subseteq \cup_{o \in S} o . \psi$ ). Note that the CoSKQ problem is to find a feasible set with the smallest cost.

Given a non-negative real number $r$, we denote the circle centered at $q . \lambda$ with radius $r$ by $C(q, r)$. Similarly, the circle centered at $o . \lambda$ with radius $r$ is denoted by $C(o, r)$.

Let $q$ be a query and $S$ be a feasible set. We say that an object $o \in S$ is a query-object distance contributor wrt $S$ if $d(o, q)$ contributes in $D_{q, o}\left(S \mid \phi_{1}\right)$. Specifically, we have the following three cases according to the value of $\phi_{1}$.

- In the case of $\phi_{1}=1$ where $D_{q, o}\left(S \mid \phi_{1}\right)=$ $\sum_{o \in S} d(o, q)$, each object in $S$ is a query-object distance contributor wrt $S$;

- In the case of $\phi_{1}=\infty$ where $D_{q, o}\left(S \mid \phi_{1}\right)=$ $\max _{o \in S} d(o, q)$, only those objects in $S$ which have the maximum distance from $q$ are the query-object distance contributors wrt $S$;

- In the case of $\phi_{1}=-\infty$ where $D_{q, o}\left(S \mid \phi_{1}\right)=$ $\min _{o \in S} d(o, q)$, only those objects in $S$ which have the minimum distance from $q$ are the query-object distance contributors wrt $S$.

Then, we define the key query-object distance contributor wrt $S$ to the object with the greatest distance from $q$ among all queryobject distance contributors wrt $S$. The concept of "key queryobject distance contributor" is inspired by the concept of "query distance owner" proposed in [17], and the concept of "key queryobject distance contributor" is more general in the sense that a query distance owner corresponds to a key query distance contributor in the case of $\phi_{1}=\infty$ but not in other cases.

Let $S$ be a set of objects and $o_{i}$ and $o_{j}$ are two objects in $S$. We say that $o_{i}$ and $o_{j}$ are object-object distance contributors wrt $S$ if $d\left(o_{i}, o_{j}\right)$ contribute in $\max _{o, o^{\prime} \in S} d\left(o, o^{\prime}\right)$, i.e, $\left(o_{i}, o_{j}\right)=$ arg $\max _{o, o^{\prime} \in S} d\left(o, o^{\prime}\right)$.

Given a query $q$ and a keyword $t$, the $t$-keyword nearest neighbor of $q$, denoted by $N N(q, t)$, it defined to be the nearest neighbor (NN) of $q$ containing keyword $t$. Similarly, $N N(o, t)$ 
is defined to be the NN of $o$ containing keyword $t$. Besides, we define the nearest neighbor set of $q$, denoted by $N(q)$ to be the set containing $q$ 's $t$-keyword nearest neighbor for each $t \in q . \psi$, i.e., $N(q)=\cup_{t \in q . \psi} N N(q, t)$. Note that $N(q)$ is a feasible set.

\subsection{An Exact Algorithm}

The idea of Unified-E is to iterate through the object-object distance contributors and search for the best feasible set $S^{\prime}$ in each iteration. This allows CoSKQ with different cost functions to be executed efficiently. Note that each existing algorithm [3], [17], [2] is designed for a specific cost function and they cannot be used to answer CoSKQ with different cost functions.

Specifically, Unified-E adopts the following search strategy.

- Step 1 (Object-Object Distance Contributors Finding): Select two objects to be the object-object distance contributors wrt the set $S^{\prime}$ to be constructed;

- $\quad$ Step 2 (Key Query-Object Distance Contributor Finding): Select an object to be the key query-object distance contributor wrt the set $S^{\prime}$ to be constructed;

- $\quad$ Step 3 (Best Feasible Set Construction): Construct the set $S^{\prime}$ (which has $o_{i}, o_{j}$ as the object-object distance contributors and $o_{m}$ as the key query-object distance contributor), and update the current best solution curSet with $S^{\prime}$ if $\operatorname{cost}\left(S^{\prime}\right)<\operatorname{curCost}$, where curCost is the cost of curSet;

- $\quad$ Step 4 (Iterative Step): Repeat Step 1 to Step 3 until all possible object-object distance contributors and key queryobject distance contributors are iterated.

The above search strategy makes quite effective pruning possible at both Step 1 and Step 2 .

Pruning at Step 1. The major idea is that not each relevant objects pair is necessary to be considered as a object-object distance contributor wrt $S^{\prime}$ to be constructed. First, only the relevant objects in $R_{S}=C\left(q, r_{1}\right)$ need to be considered, where $r_{1}$ is the radius of the region that depends on the parameter setting, as shown in Table 2. It can be proved that if $S^{\prime}$ contains an object $O$ such that $d(o, q)>r_{1}, S^{\prime}$ cannot be the optimal solution. Second, we can maintain a lower bound $d_{L B}$ and an upper bound $d_{U B}$ of the distance between the object-object distance contributors for pruning. For example, all those relevant objects pairs $\left(o_{i}, o_{j}\right)$ with $d\left(o_{i}, o_{j}\right)>$ curCost (this is because in this case, all those feasible sets $S^{\prime}$ with $\left(o_{i}, o_{j}\right)$ as the object-object distance contributor have the cost larger than that of the current best solution, i.e., the best-known cost) could be pruned, i.e., curCost is used as an upper bound. Furthermore, it could be verified easily that when $\phi_{1} \in\{1, \infty\}$, all those relevant object pairs $\left(o_{i}, o_{j}\right)$ with $d\left(o_{i}, o_{j}\right)<\max _{o \in N(q)} d(o, q)-\min \left\{d\left(o_{i}, q\right), d\left(o_{j}, q\right)\right\}$ could be pruned, i.e., $\max _{o \in N(q)} d(o, q)-\min \left\{d\left(o_{i}, q\right), d\left(o_{j}, q\right)\right\}$ is used as a lower bound. The details of $d_{L B}$ and $d_{U B}$ for different parameter settings are presented in Table 2 Specifically, we have the following lemma.

Lemma 1. Let $o_{i}$ and $o_{j}$ be the object-object distance contributors of the set $S$ to be constructed. For cost unified $_{\text {with different }}$ parameter settings, $d\left(o_{i}, o_{j}\right)$ can be lower bounded by $d_{L B}$ and upper bounded by $d_{U B}$, as shown in Table 2

Proof. Let $o_{m}$ be the key query-object distance contributor of $S$. The proof of $d_{L B}$ is shown as follows. When $\phi_{1} \in\{1, \infty\}$, $d\left(o_{i}, o_{j}\right) \geq d\left(o_{i}, o_{m}\right)$ and $d\left(o_{i}, o_{j}\right) \geq d\left(o_{j}, o_{m}\right)$. Besides, we know that $d\left(o_{i}, o_{m}\right)+d\left(o_{i}, q\right) \geq d\left(o_{m}, q\right)$ by triangle inequality. Similarly, we know that $d\left(o_{j}, o_{m}\right)+d\left(o_{j}, q\right) \geq$ $d\left(o_{m}, q\right)$. Since $S$ is feasible, $d\left(o_{m}, q\right) \geq d_{f}$. Therefore, we have $d\left(o_{i}, o_{j}\right) \geq d\left(o_{m}, q\right)-\min \left\{d\left(o_{i}, q\right), d\left(o_{j}, q\right)\right\} \geq d_{f}-$ $\min \left\{d\left(o_{i}, q\right), d\left(o_{j}, q\right)\right\}=d_{L B}$. When $\phi_{1}=-\infty$, we have $d\left(o_{m}, q\right)+d\left(o_{i}, o_{j}\right) \geq d_{f}$ because $S$ is feasible. Also, $d\left(o_{i}, q\right) \geq$ $d\left(o_{m}, q\right)$ and $d\left(o_{j}, q\right) \geq d\left(o_{m}, q\right)$ because $o_{m}$ is the object closet to $q$. Therefore, we have $d\left(o_{i}, o_{j}\right) \geq d_{f}-d\left(o_{m}, q\right) \geq d_{f}-$ $\min \left\{d\left(o_{i}, q\right), d\left(o_{j}, q\right)\right\}=d_{L B}$.

The proof of $d_{U B}$ is shown as follows. When $\alpha=0.5, \phi_{1}=1$ and $\phi_{2}=1\left(\right.$ cost $\left._{\text {SumMax }}\right), \operatorname{cost}(S) \geq d\left(o_{i}, q\right)+d\left(o_{j}, q\right)+$ $d\left(o_{i}, o_{j}\right)$ and $d\left(o_{i}, q\right)+d\left(o_{j}, q\right) \geq d\left(o_{i}, o_{j}\right)$ by triangle inequality. If $d\left(o_{i}, o_{j}\right) \geq \operatorname{curCost} / 2$, we have $\operatorname{cost}(S) \geq 2 d\left(o_{i}, o_{j}\right) \geq$ curCost, which means $S$ cannot contribute to a better solution and can be pruned. When $\alpha=0.5, \phi_{1}=\infty$ and $\phi_{2}=1$ $\left(\operatorname{cost}_{\text {MaxMax }}\right), \operatorname{cost}(S)=d\left(o_{m}, q\right)+d\left(o_{i}, o_{j}\right)$ and $d\left(o_{m}, q\right) \geq$ $d_{f}$ since $S$ is a feasible set. If $d\left(o_{i}, o_{j}\right) \geq$ curCost $-d_{f}$, we have $\operatorname{cost}(S) \geq$ curCost and thus $S$ can be pruned. For the other parameter settings, it is easy to see that if $S$ contain an object $O$ with $d(o, q) \geq$ curCost, $\operatorname{cost}(S) \geq$ curCost.

Third, given a set having $o_{i}$ and $o_{j}$ as the object-object distance contributors, we can compute the lower bound of cost of the set, denoted by $\operatorname{cost}\left(\left\{o_{i}, o_{j}\right\}\right)_{L B}$, and thus we can prune all those object pairs with $\operatorname{cost}\left(\left\{o_{i}, o_{j}\right\}\right)_{L B}>$ curCost. The details of $\operatorname{cost}\left(\left\{o_{i}, o_{j}\right\}\right)_{L B}$ for different parameter settings are presented in Table 2 Specifically, we have the following lemma.

Lemma 2. Let $o_{i}$ and $o_{j}$ be the object-object distance contributors of the set $S$ to be constructed. For cost $t_{\text {unified }}$ with different parameter settings, $\operatorname{cost}(S)$ can be lower bounded by $\operatorname{cost}\left(\left\{o_{i}, o_{j}\right\}\right)_{L B}$, as shown in Table 2

Proof. Let $o_{m}$ be the key query-object distance contributor of $S$. When $\phi_{1}=1$, it is obvious that $\operatorname{cost}(S) \geq \operatorname{cost}\left(\left\{o_{i}, o_{j}\right\}\right)_{L B}$.

When $\phi_{1}=\infty, d\left(o_{m}, q\right) \geq \max \left\{d\left(o_{i}, q\right), d\left(o_{j}, q\right)\right\}$. Since $S$ is a feasible set, $d\left(o_{m}, q\right) \geq d_{f}$. Thus, $\operatorname{cost}(S) \geq d\left(o_{i}, o_{j}\right)+$ $\max \left\{d\left(o_{i}, q\right), d\left(o_{j}, q\right), d_{f}\right\}$ when $\phi_{2}=1\left(\operatorname{cost}_{\text {MaxMax }}\right)$ and $\operatorname{cost}(S) \geq \max \left\{d\left(o_{i}, o_{j}\right), d\left(o_{i}, q\right), d\left(o_{j}, q\right), d_{f}\right\}$ when $\phi_{2}=\infty$ $\left(\operatorname{cost}_{\operatorname{Max} \operatorname{Max} 2}\right)$.

When $\phi_{1}=-\infty$ and $\phi_{2}=1\left(\operatorname{cost}_{\text {MinMax }}\right)$, we know that $\operatorname{cost}(S) \geq d\left(o_{i}, o_{j}\right)$. Also we have $\operatorname{cost}(S) \geq d\left(o_{m}, q\right)+$ $d\left(o_{i}, o_{m}\right) \geq d\left(o_{i}, q\right)$ by triangle inequality. Similarly, we have $\operatorname{cost}(S) \geq d\left(o_{m}, q\right)+d\left(o_{j}, o_{m}\right) \geq d\left(o_{j}, q\right)$. Therefore, $\operatorname{cost}(S) \geq \max \left\{d\left(o_{i}, o_{j}\right), d\left(o_{i}, q\right), d\left(o_{j}, q\right)\right\}$.

When $\phi_{1}=-\infty$ and $\phi_{2}=\infty\left(\right.$ cost $\left._{\text {MinMax } 2}\right)$, we know that $\operatorname{cost}(S) \geq d\left(o_{i}, o_{j}\right)$. Also $d\left(o_{m}, q\right) \geq d\left(o_{i}, q\right)-d\left(o_{i}, o_{j}\right)$ because $o_{m}$ must be located in the region of $C\left(o_{i}, d\left(o_{i}, o_{j}\right)\right)$. Similarly, $d\left(o_{m}, q\right) \geq d\left(o_{j}, q\right)-d\left(o_{i}, o_{j}\right)$. Therefore, $\operatorname{cost}(S) \geq$ $\max \left\{d\left(o_{i}, o_{j}\right), \max \left\{d\left(o_{i}, q\right), d\left(o_{j}, q\right)\right\}-d\left(o_{i}, o_{j}\right)\right\}$.

Pruning at Step 2. Note that only the objects in $C\left(o_{i}, d\left(o_{i}, o_{j}\right)\right) \cap$ $C\left(o_{j}, d\left(o_{i}, o_{j}\right)\right)$ need to be considered as key query-object distance contributors for constructing $S^{\prime}$. The major idea of the pruning is that not all possible objects in the region are necessary to be considered. Specifically, we can maintain a lower bound $r_{L B}$ and an upper bound $r_{U B}$ of the distance between the key query-object distance contributors and query. For example, in the case that $\phi_{1}=1$, all those relevant objects $o$ with $d(o, q)<\max \left\{d\left(o_{i}, q\right), d\left(o_{j}, q\right)\right\}$ could be safely pruned (this is because such object $O$ can not be the key query-object distance contributor wrt $\left.S^{\prime}\right)$, i.e., $\max \left\{d\left(o_{i}, q\right), d\left(o_{j}, q\right)\right\}$ is used as lower bound. Figure 1 (a) shows the region for the objects to be considered as the key query-object distance contributor. In the 


\begin{tabular}{|c|c|c|c|c|c|c|c|}
\hline \multirow{2}{*}{ Cost function } & \multicolumn{3}{|c|}{ Parameter } & \multirow{2}{*}{$r_{1}$} & \multirow{2}{*}{$d_{L B}$} & \multirow{2}{*}{$d_{U B}$} & \multirow{2}{*}{$\operatorname{cost}\left(\left\{o_{i}, o_{j}\right\}\right)_{L B}$} \\
\hline & $\alpha$ & $\phi_{1}$ & $\phi_{2}$ & & & & \\
\hline $\operatorname{cost}_{M a x M a x}$ & 0.5 & $\infty$ & 1 & curCost & \multirow{4}{*}{$d_{f}-\min \left\{d\left(o_{i}, q\right), d\left(o_{j}, q\right)\right\}$} & curCost $-d_{f}$ & $d\left(o_{i}, o_{j}\right)+\max \left\{d\left(o_{i}, q\right), d\left(o_{j}, q\right), d_{f}\right\}$ \\
\hline cost $_{\text {MinMax }}$ & 0.5 & $-\infty$ & 1 & curCost & & curCost & $\max \left\{d\left(o_{i}, o_{j}\right), d\left(o_{i}, q\right), d\left(o_{j}, q\right), d_{f}\right\}$ \\
\hline cost $_{\text {MinMax } 2}$ & 0.5 & $-\infty$ & $\infty$ & $2 \cdot$ curCost & & curCost & $\max \left\{d\left(o_{i}, o_{j}\right), \max \left\{d\left(o_{i}, q\right), d\left(o_{j}, q\right)\right\}-d\left(o_{i}, o_{j}\right)\right\}$ \\
\hline cost $_{\text {Sum }}$ & 1 & 1 & - & curCost & & curCost & $d\left(o_{i}, q\right)+d\left(o_{j}, q\right)$ \\
\hline
\end{tabular}

$d_{f}=\max _{o \in N(q)} d(o, q) \quad$ TABLE 2: Lower and upper bounds used in Step 1 of Unified-E

\begin{tabular}{|c|c|c|c|c|c|}
\hline \multirow{2}{*}{ Cost function } & \multicolumn{3}{|c|}{ Parameter } & \multirow{2}{*}{$r_{L B}$} & \multirow{2}{*}{$r_{U B}$} \\
\hline & $\alpha$ & $\phi_{1}$ & $\phi_{2}$ & & \\
\hline cost $_{\text {SumMax }}$ & 0.5 & 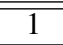 & 1 & $\max \left\{d\left(o_{i}, q\right), d\left(o_{j}, q\right), d_{f}\right\}$ & $\overline{c u r C o s t-d\left(o_{i}, o_{j}\right)}$ \\
\hline $\operatorname{cost}_{\text {MaxMax }}$ & 0.5 & $\infty$ & 1 & $\max \left\{d\left(o_{i}, q\right), d\left(o_{j}, q\right), d_{f}\right\}$ & curCost $-d\left(o_{i}, o_{j}\right)$ \\
\hline $\operatorname{cost}_{\operatorname{Max} \operatorname{Max2}}$ & 0.5 & $\infty$ & $\infty$ & $\max \left\{d\left(o_{i}, o_{j}\right), d_{f}\right\}$ & curCost \\
\hline cost $_{\text {MinMax }}$ & 0.5 & $-\infty$ & 1 & $d_{f}-d\left(o_{i}, o_{j}\right)$ & $\min \left\{\operatorname{curCost}-d\left(o_{i}, o_{j}\right), \min \left\{d\left(o_{i}, q\right), d\left(o_{j}, q\right)\right\}\right\}$ \\
\hline cost $_{\text {MinMax } 2}$ & 0.5 & $-\infty$ & $\infty$ & $d_{f}-d\left(o_{i}, o_{j}\right)$ & $\min \left\{d\left(o_{i}, q\right), d\left(o_{j}, q\right)\right\}$ \\
\hline cost $_{\text {Sum }}$ & 1 & 1 & - & $\max \left\{d\left(o_{i}, q\right), d\left(o_{j}, q\right), d_{f}\right\}$ & curCost $-d\left(o_{i}, q\right)-d\left(o_{j}, q\right)$ \\
\hline
\end{tabular}

TABLE 3: Lower and upper bounds used in Step 2 of Unified-E

case that $\phi_{1}=-\infty$, similarly, all those relevant objects $o$ with $d(o, q)>\min \left\{d\left(o_{i}, q\right), d\left(o_{j}, q\right)\right\}$ could be safely pruned i.e., $\min \left\{d\left(o_{i}, q\right), d\left(o_{j}, q\right)\right\}$ is used as upper bound. Also, all those relevant objects $o$ with $d(o, q)<d_{f}-d\left(o_{i}, o_{j}\right)$ could be safely pruned, where $d_{f}=\max _{o \in N(q)} d(o, q)$ (this is because all those feasible sets $S^{\prime}$ with $O$ as the key query-object distance contributor have $\max _{o_{1}, o_{2} \in S^{\prime}} d\left(o_{1}, o_{2}\right)$ larger than $\left.d\left(o_{i}, o_{j}\right)\right)$, i.e., $d_{f}-d\left(o_{i}, o_{j}\right)$ is used as an lower bound. Figure 1 (b) shows the region for the objects to be considered as the key query-object distance contributor. The details of $r_{L B}$ and $r_{U B}$ for different parameter settings are presented in Table 3

Specifically, we have the following lemma.

Lemma 3. Let $o_{i}$ and $o_{j}$ be the object-object distance contributors and $o_{m}$ be the key query-object distance contributors of the set $S$ to be constructed. For cost $t_{\text {unified }}$ with different parameter settings, $d\left(o_{m}, q\right)$ can be lower bounded by $r_{L B}$ and upper bounded by $r_{U B}$, as shown in Table 3

Proof. The proof of $r_{L B}$ is shown as follows. When $\phi_{1} \in\{1, \infty\}$, $d\left(o_{m}, q\right)>d_{f}$ because otherwise $S$ is not a feasible set. For $\operatorname{cost}_{\text {SumMax }}, \operatorname{cost}_{\text {MaxMax }}$ and $\operatorname{cost}_{\text {Sum }}$, we do not need to consider an object $o$ if $d(o, q)<\max \left\{d\left(o_{i}, q\right), d\left(o_{j}, q\right)\right\}$ because it can not be the key query-object distance contributor of $S$ by definition. Similarly, for $\operatorname{cost}_{\operatorname{Max} M a x 2}$, we do not need to consider object $o$ if $d(o, q)<d\left(o_{i}, o_{j}\right)$ because it cannot be the key query-object distance contributor of $S$. When $\phi_{1}=-\infty$, we set $r_{L B}=d_{f}-d\left(o_{i}, o_{j}\right)$ because otherwise $S$ is not a feasible set.

The proof of $r_{U B}$ is shown as follows. For cost $\operatorname{sumMax}_{\text {Sum }}$ and cost $_{\text {MaxMax }}$, if $S$ contains an object $o$ with $d(o, q) \geq$ curCost $-d\left(o_{i}, o_{j}\right)$, it is obvious that $\operatorname{cost}(S) \geq \operatorname{curCost}$. Similarly, for $\operatorname{cost}_{\text {MaxMax } 2}\left(\right.$ cost $\left._{S u m}\right)$, if $S$ contains an object $o$ with $d(o, q) \geq \operatorname{curCost}(d(o, q)>$ curCost $\left.d\left(o_{i}, q\right)-d\left(o_{j}, q\right)\right), \operatorname{cost}(S) \geq \operatorname{curCost}$. For cost $_{\text {MinMax }}$ and cost $_{\text {MinMax } 2}$, we do not need to consider an object $o$ if $d(o, q) \geq$ $\min \left\{d\left(o_{i}, q\right), d\left(o_{j}, q\right)\right.$ because it can not be the key query-object distance contributor of $S$ by definition. Also, in cost $_{\text {MinMax }}$, if $d(o, q) \geq \operatorname{curCost}-d\left(o_{i}, o_{j}\right), \operatorname{cost}(S) \geq$ curCost .

With the above search strategy introduced, we present the Unified-E algorithm in Algorithm 1. Specifically, we maintain an object set curSet for storing the best-known solution found so far, which is initialized to $N(q)$ (line 1), and curCost to be the

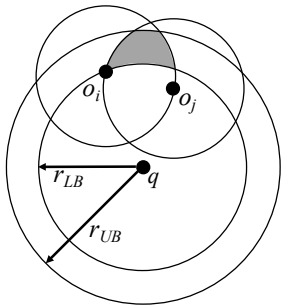

(a) $\phi_{1} \in\{1, \infty\}$

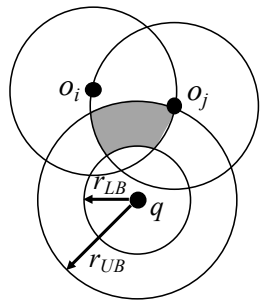

(b) $\phi_{1}=-\infty$
Fig. 1: Pruning at Step 2 of Unified-E

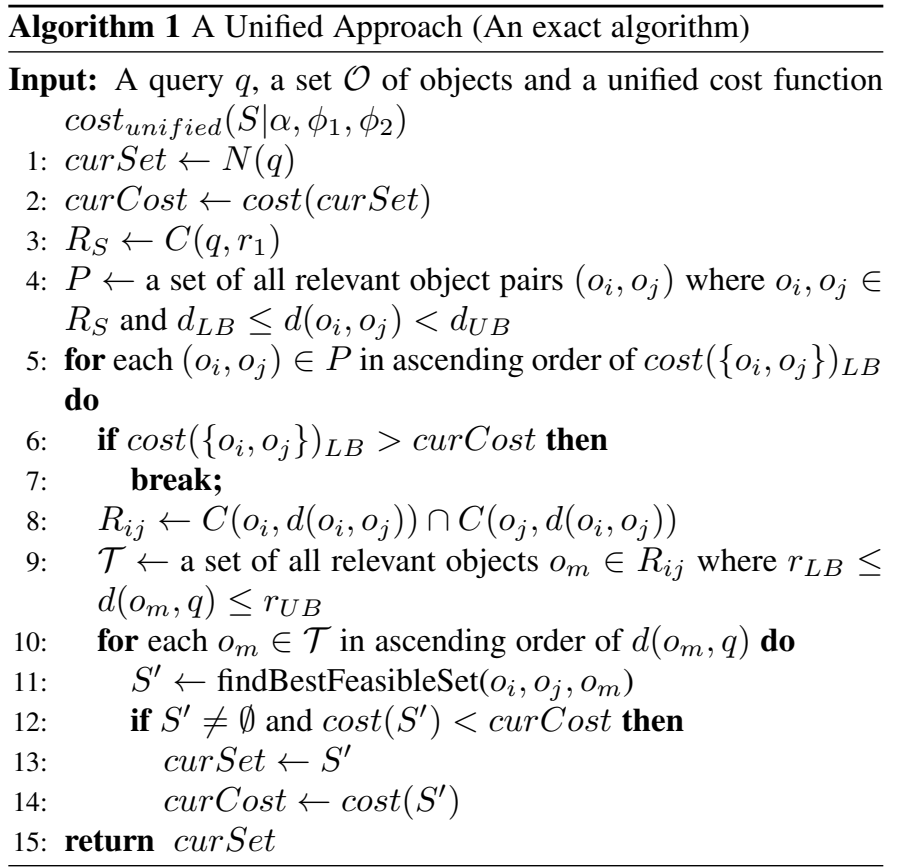

cost of curSet (line 2). Recall that $N(q)$ is a feasible set. Then, we initialize $R_{S}$ to be $C\left(q, r_{1}\right)$ (line 3 ) and find a set $P$ of all object pairs $\left(o_{i}, o_{j}\right)$ where $o_{i}$ and $o_{j}$ are in $R_{S}$ to take the roles of object-object distance contributors (line 4).

Second, we perform an iterative process as follows. Consider one iteration. We check whether the lower bound of the set containing $o_{i}$ and $o_{j}$ is larger than curCost (line 6). If yes, we stop the iterations (line 7). Otherwise, we proceed to initialize the re- 


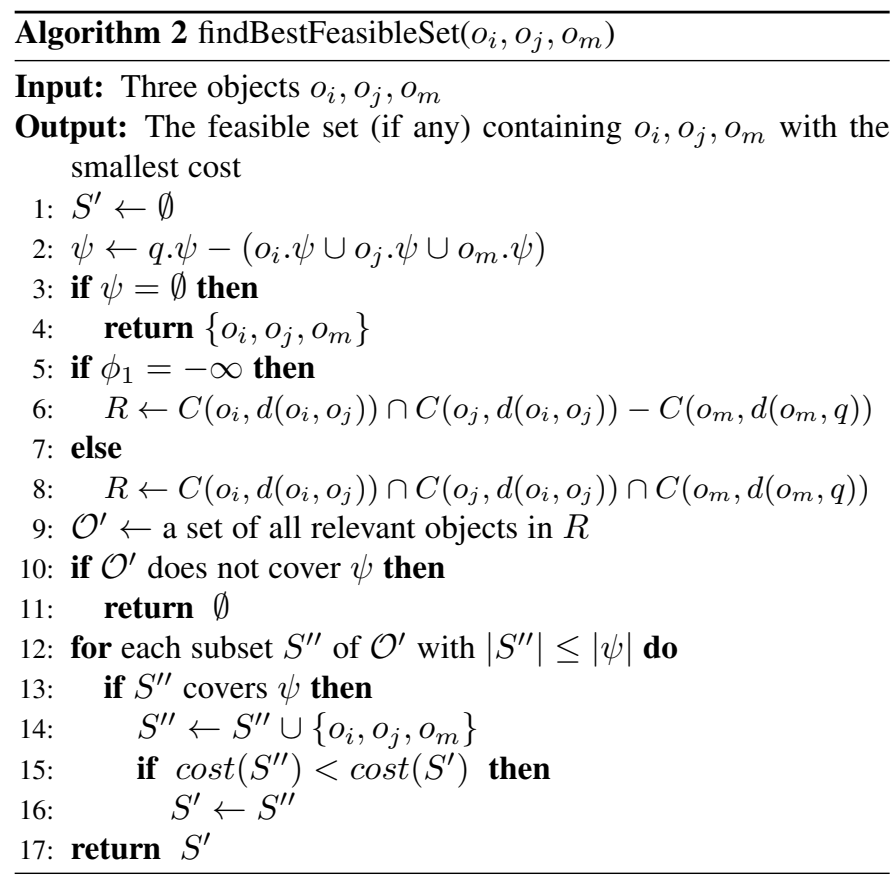

gion $R_{i j}$ to $C\left(o_{i}, d\left(o_{i}, o_{j}\right)\right) \cap C\left(o_{j}, d\left(o_{i}, o_{j}\right)\right)$ (line 8) and find a set $\mathcal{T}$ of all objects $o_{m}$ where $o_{m}$ is in $R_{i j}$ to take the role of key query-object distance contributor (line 9 ).

Third, we invoke a procedure called findBestFeasibleSet (discussed later) for constructing a feasible set $S^{\prime}$ which takes $o_{i}$ and $o_{j}$ as the object-object distance contributors and $o_{m}$ as the key query-object distance contributor wrt $S^{\prime}$ (line 11). Then, we update curSet to $S^{\prime}$ if $S^{\prime}$ exists and $\operatorname{cost}\left(S^{\prime}\right)<\operatorname{curCost}$ (lines 12 - 14).

Fourth, we iterate the process with the next relevant object in $R_{i j}$ and with the next object pair from $R_{S}$ until all relevant objects in $R_{S}$ have been processed.

Next, we introduce the "findBestFeasibleSet" procedure (used in Algorithm 11, which takes three objects $o_{i}, o_{j}$ and $o_{m}$ as input and finds the best feasible set $S^{\prime}$ (if any) with the smallest cost among all feasible sets which have $o_{i}$ and $o_{j}$ as the object-object distance contributors have $o_{m}$ as a key query-object distance contributor. The procedure is presented in Algorithm 2 and it works as follows. First, it initializes $S^{\prime}$ as an empty set (line 1). Then, it initializes a variable $\psi$, denoting the set of keywords in $q . \psi$ not covered by $S^{\prime}$ yet, as $q . \psi-\left(o_{i} . \psi \cup o_{j} . \psi \cup o_{m} . \psi\right)$ (line 2). If $\psi=\emptyset$, then it returns $\left\{o_{i}, o_{j}, o_{m}\right\}$ immediately (lines 3-4). Otherwise, it proceeds to retrieve the set $\mathcal{O}^{\prime}$ containing all relevant objects in $R$, where $R$ is defined based on the value of $\phi_{1}$ (lines 5-9). When $\phi_{1} \in\{1, \infty\}, R=C\left(o_{i}, d\left(o_{i}, o_{j}\right)\right) \cap C\left(o_{j}, d\left(o_{i}, o_{j}\right)\right) \cap$ $C\left(o_{m}, d\left(o_{m}, q\right)\right)$ (line 6), and the region is shown in Figure 2 a). When $\phi_{1}=-\infty, R=C\left(o_{i}, d\left(o_{i}, o_{j}\right)\right) \cap C\left(o_{j}, d\left(o_{i}, o_{j}\right)\right)-$ $C\left(o_{m}, d\left(o_{m}, q\right)\right)$ (line 8), and the region is shown in Figure 2 (b). The major idea of the region $R$ is that including any object outside the region would violate one or both of the following constraints: (1) $o_{m}$ is the key query-object distance contributor of the set to be found and (2) $o_{i}$ and $o_{j}$ are the object-object distance contributors of the set to be found. If $\mathcal{O}^{\prime}$ does not cover $\psi$, it returns $\emptyset$ immediately which implies that no such feasible set could be found (lines 10-11). Otherwise, it finds the target by enumerating all possible subsets $S^{\prime \prime}$ of $\mathcal{O}^{\prime}$ with size at most $|\psi|$ (by utilizing the inverted lists maintained for each keyword in $\psi$ ), and for each possible $S^{\prime \prime}$, if it covers $\psi$ and $\operatorname{cost}\left(S^{\prime \prime} \cup\left\{o_{i}, o_{j}, o_{m}\right\}\right)<\operatorname{cost}\left(S^{\prime}\right), S^{\prime}$ is

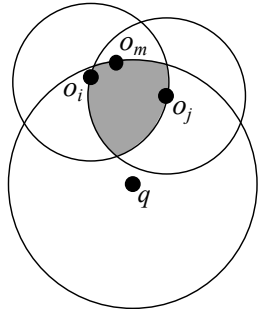

(a) $\phi_{1} \in\{1, \infty\}$

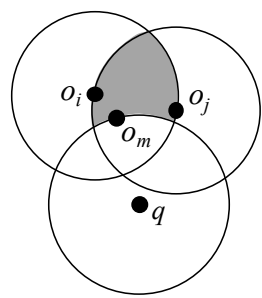

(b) $\phi_{1}=-\infty$
Fig. 2: Search space $R$ in Algorithm 2

updated correspondingly (lines 12-16).

We also develop some other pruning techniques based on a concept of "dominance" for further improving the efficiency of the algorithm. The major idea is that under some parameter settings, the solution of the CoSKQ problem contains only those objects that are not dominated by other objects. Details could be found in Appendix C

Time complexity analysis. Let $|P|$ be the number of object pairs in $P$. Note that $|P|$ is usually much smaller than $\left|\mathcal{O}_{q}\right|^{2}$ since $|P|$ corresponds to the number of relevant objects we process in $R_{S}$ and the area occupied by $R_{S}$ is typically small. Let $\left|R_{i j}\right|$ be the number of relevant objects in $R_{i j}$. The time complexity of Algorithm 1 is $O\left(|P| \cdot\left|R_{i j}\right| \cdot \theta\right)$, where $\theta$ is the time complexity of Algorithm 2. It could be verified that $\theta$ is dominated by the step of enumerating the object sets (lines 12-16 in Algorithm 2), whose cost is $O\left(\left.\left|\mathcal{O}^{\prime}\right| q \cdot \psi|-3 \cdot| \psi\right|^{2}\right)$ since it searches at most $O\left(\left|\mathcal{O}^{\prime}\right| q . \psi \mid-3\right)$ subsets $S^{\prime \prime}$ that cover $\psi$ and the checking cost for each subset is $O\left(|\psi|^{2}\right)$. As a result, the time complexity of Unified-E is $O\left(\left.|P| \cdot\left|R_{i j}\right| \cdot\left|\mathcal{O}^{\prime}\right| q \cdot \psi|-3 \cdot| \psi\right|^{2}\right)$.

\subsection{An Approximate Algorithm}

In this part, we introduce the approximate algorithm Unified-A. Compared with Unified-E, Unified-A drops the step of objectobject distance contributors finding and replaces the step of best feasible set construction which is expensive with a step of (arbitrary) feasible set construction which is efficient, and thus it enjoys significantly better efficiency. Specifically, the Unified-A adopts the following search strategy.

- Step 1 (Key Query-Object Distance Contributor Finding): Select a relevant object $o$ to be key query-object distance contributor wrt a set $S^{\prime}$ to be constructed;

- Step 2 (Feasible Set Construction): Construct the set $S^{\prime}$ (which has $O$ as a key query-object distance contributor);

- Step 3 (Optimal Set Updating): Update the current best solution curSet if $\operatorname{cost}\left(S^{\prime}\right)<$ curCost, where curCost is the cost of curSet;

- Step 4 (Iterative Step): Repeat Step 1 to Step 3 until all possible key query-object distance contributors are iterated.

The above search strategy makes quite effective pruning possible at both Step 1 and Step 2.

Pruning at Step 1. The major idea is that not each relevant object is necessary to be considered as a key query-object distance contributor wrt $S^{\prime}$ to be constructed. Specifically, in the case of $\phi_{1} \in$ $\{1, \infty\}$, all those relevant objects $o$ with $d(o, q)>$ curCost (this is because all those feasible sets $S^{\prime}$ with $o$ as a key queryobject distance contributor have the cost larger than the bestknown cost curCost, and thus they could be pruned) or $d(o, q)<$ $\max _{o \in N(q) d(o, q)}$ (this is because there exist no feasible sets within the disk of $C\left(q, \max _{o \in N(q)} d(o, q)-\epsilon\right)$ where $\epsilon$ is close to zero) 


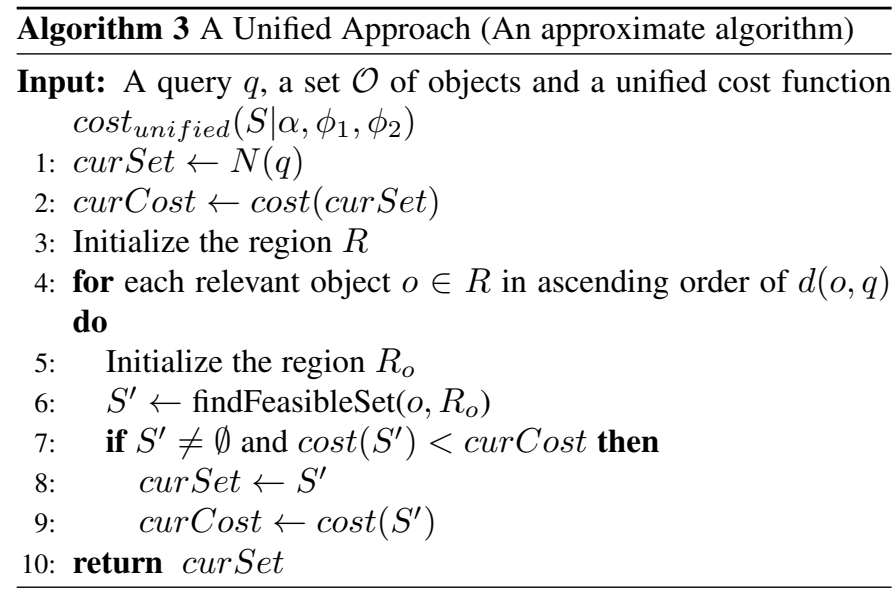

could be pruned. Therefore, we can maintain a region $R$ which corresponds to the "ring region" enclosed by $C(q$, curCost $)$ and $C\left(q, \max _{o \in N}(q) d(o, q)\right)$ for pruning the search space at Step 1. In the case of $\phi_{1}=-\infty$, the region $R$ could also be defined correspondingly. Details of the region $R$ for different parameter settings are presented in Table 4 .

Pruning at Step 2. We define a region $R_{o}$ by the key query-object distance contributor found in Step 1 and only the objects in the region need to be considered for constructing $S^{\prime}$. The major idea of the pruning is that not all possible objects in $R_{o}$ are necessary to be considered. Specifically, in the case of $\phi_{1} \in\{1, \infty\}$, all those relevant objects outside $C(q, d(o, q))$ could be safely pruned (this is because including one such object would fail $o$ to be a key query-object distance contributor wrt $S^{\prime}$ ). Thus, we can maintain a region $R_{o}$ which corresponds to $C(q, d(o, q))$ for pruning the search space at Step 2. In the case of $\phi_{1}=-\infty$, the region $R_{o}$ could also be maintained appropriately. Details of the region $R_{o}$ for different parameter settings are presented in Table 4 as well.

With the above search strategy and pruning techniques introduced, the Unified-A algorithm is presented in Algorithm 3 Specifically, we maintain an object set cur Set for storing the bestknown solution found so far, which is initialized to $N(q)$ (line 1) and curCost to be the cost of curSet (line 2). Then, we perform an iterative process for each relevant object $o \in R$ in ascending order of $d(o, q)$ (lines 3-4). Consider one iteration. First, we initialize the region $R_{o}$ (line 5). Second, we invoke a procedure called findFeasibleSet (discussed later) for constructing a feasible set $S^{\prime}$ which takes $o$ as a key query-object distance contributor wrt $S^{\prime}$ (line 6). Third, we update curSet to $S^{\prime}$ and curCost to $\operatorname{cost}\left(S^{\prime}\right)$ if $S^{\prime}$ exists and $\operatorname{cost}\left(S^{\prime}\right)<\operatorname{curCost}$ (lines 7-9). We iterate the process with the next relevant object from $R$ which has not been processed until all relevant objects in $R$ have been processed.

Next, we introduce the "findFeasibleSet" procedure (used in Algorithm 3, which takes an object $o$ and a region $R_{o}$ as input and finds a feasible set $S^{\prime}$ (if any) which contains objects in $R_{o}$ (including $o$ ) and has $o$ as a key query-object distance contributor. The procedure is presented in in Algorithm 4, and it is similar to the "findBestFeasibleSet" procedure (in Algorithm 2) except that it replaces the enumeration process with an iterative process (lines 8-14) for searching for a feasible set.

Depending on the value of $\phi_{1}$, the algorithm uses different criterion for picking an object at an iteration, which is described as follows.

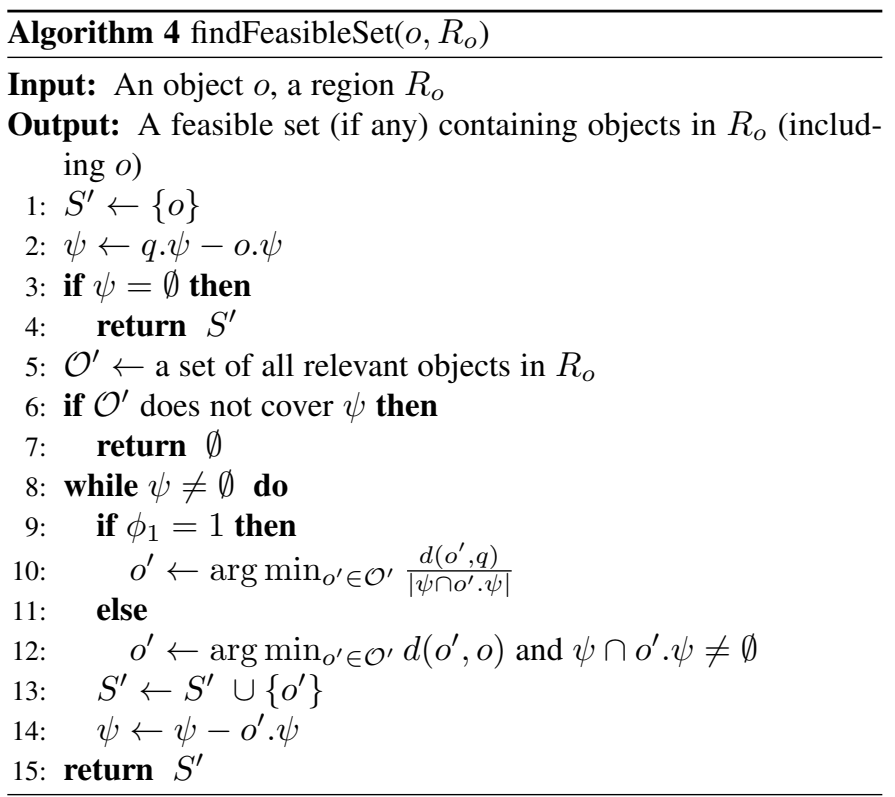

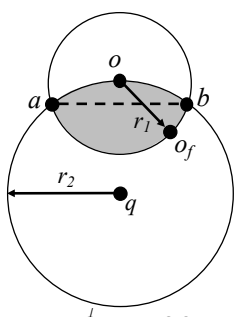

(a) $\begin{aligned} & \phi_{1}=\infty \\ & r_{1} \leq \sqrt{2} r_{2}\end{aligned}$

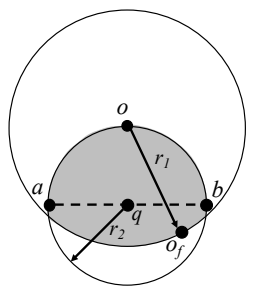

(b) $\phi_{1}=\infty$,

$\phi_{1}=\infty$
$r_{1}>\sqrt{2} r_{2}$

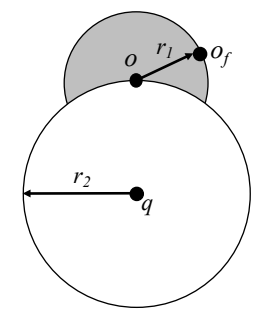

(c) $\phi_{1}=-\infty$
Fig. 3: Illustration of proof of Theorem 2

Case 1: $\phi_{1}=1$. It picks the object which has the smallest ratio of its distance to $q$ to the number of remaining keywords covered. Using this criterion, the algorithm tries to pick objects in a way that minimizes the sum of the distances between the query location and the objects.

Case 2: $\phi_{1} \in\{\infty,-\infty\}$. It picks the object which is the nearest to $O$ and covers some of the uncovered keywords. Using this criterion, the algorithm tries to pick objects in a way that minimizes the maximum pairwise distance between the objects.

We also develop two techniques based on the concept of information re-use for implementing the Unified-A with better efficiency. The details could be found in Appendix D

Time complexity analysis. Let $|R|$ be the number of relevant objects in $R$. It could be verified that the complexity of the "findFeasibleSet" (Algorithm 4) is $O\left(|\psi| \cdot\left|\mathcal{O}^{\prime}\right| \log \left|\mathcal{O}^{\prime}\right|\right.$ ) (note that a heap structure with $\left|\mathcal{O}^{\prime}\right|$ elements could be used and there are at most $O(|\psi|)$ operations based on the heap). Therefore, the time complexity of Unified-A is $O\left(|R| \cdot|\psi| \cdot\left|\mathcal{O}^{\prime}\right| \log \left|\mathcal{O}^{\prime}\right|\right)$.

Approximation ratio analysis. In general, the Unified-A algorithm gives different approximation ratios for different parameter settings, which are given in the following theorem.

Theorem 2. The Unified-A algorithm gives approximation ratios as shown in Table 5 for the CoSKQ problem under different parameter settings.

Proof. Let $O$ be the key query-object distance contributor wrt the optimal solution $S_{o}$. Let $S$ be the solution returned by Unified-A. In the following, we analyze the approximation ratio of $\operatorname{cost}(S) / \operatorname{cost}\left(S_{o}\right)$ with different parameter settings. 


\begin{tabular}{|c|c|c|c|c|c|}
\hline \multirow{2}{*}{ Cost function } & \multicolumn{3}{|c|}{ Parameter } & \multirow{2}{*}{$R$} & \multirow{2}{*}{$R_{o}$} \\
\hline & $\alpha$ & $\phi_{1}$ & $\phi_{2}$ & & \\
\hline cost $_{\text {SumMax }}$ & 0.5 & $\overline{1} 1$ & 1 & $C(q$, curCost $)-C\left(q, d_{f}\right)$ & $C(q, d(o, q))$ \\
\hline cost $_{\text {MaxMax }}$ & 0.5 & $\infty$ & 1 & $C(q$, curCost $)-C\left(q, d_{f}\right)$ & $C(q, d(o, q))$ \\
\hline $\operatorname{cost}_{\operatorname{Max} \operatorname{Max} 2}$ & 0.5 & $\infty$ & $\infty$ & $C(q$, curCost $)-C\left(q, d_{f}\right)$ & $C(q, d(o, q))$ \\
\hline $\operatorname{cost}_{\text {MinMax }}$ & 0.5 & $-\infty$ & 1 & $C(q$, curCost $)$ & $C(q$, curCost $) \cap C(o$, curCost $-d(o, q))-C(q, d(o, q))$ \\
\hline cost $_{\operatorname{MinMax} 2}$ & 0.5 & $-\infty$ & $\infty$ & $C(q, 2 \cdot \operatorname{curCost})$ & $C(q, 2 \cdot$ curCost $) \cap C(o$, curCost $-d(o, q))-C(q, d(o, q))$ \\
\hline cost $_{\text {Sum }}$ & 1 & 1 & - & $C(q$, curCost $)-C\left(q, d_{f}\right)$ & $C(q, d(o, q))$ \\
\hline
\end{tabular}

TABLE 4: $R$ and $R_{o}$ in Unified-A

The algorithm iterates each object in the region $R$, and from the way we initialize $R$, there must exists an iteration in Unified-A such that it processes $O$ and thus it finds the corresponding feasible set $S^{\prime}$. Note that $o$ is the key query-object distance contributor of $S^{\prime}$ because the other objects in $S^{\prime}$ are located in the region $R_{o}$. We have $\operatorname{cost}(S) \leq \operatorname{cost}\left(S^{\prime}\right)$ because Unified-A returns the feasible set with the smallest cost. The following proof shows that $\operatorname{cost}\left(S^{\prime}\right) \leq \gamma \operatorname{cost}\left(S_{o}\right)$ which further implies $\operatorname{cost}(S) \leq$ $\gamma \operatorname{cost}\left(S_{o}\right)$, where $\gamma$ is the approximation ratio. We consider three cases based on the values of $\phi_{1}$ as follows.

Case 1. $\phi_{1}=1$. In this case, the approach of the algorithm to pick object to form $S^{\prime}$ is modified from the approximation algorithm of the Weighted Set Cover (WSC) problem, where the keywords in $\psi$ correspond to elements, the objects correspond to sets, and the distances between the objects and query correspond to the set costs. The proof is based on the approximation properties of the WSC problem. Let $w^{\prime}=\sum_{o^{\prime} \in S^{\prime} \backslash\{o\}} d\left(o^{\prime}, q\right)$ and $w_{o}=$ $\sum_{o^{\prime} \in S_{o} \backslash\{o\}} d\left(o^{\prime}, q\right)$. We have $w^{\prime} \leq H_{|\psi|} w_{o}$ where $\psi=q . \psi-$ $o . \psi,|\psi|<|q . \psi|$ and $H_{k}$ is the $k^{t h}$ harmonic number. There are three parameter settings (cost functions) adopt this picking object criterion, the proof are shown as follows.

Case 1(a). $\alpha=1, \phi_{1}=1$ ( cost $\left._{\text {Sum }}\right)$.

$$
\begin{aligned}
\frac{\operatorname{cost}\left(S^{\prime} \mid 1,1, \cdot\right)}{\operatorname{cost}\left(S_{o} \mid 1,1, \cdot\right)} & =\frac{\sum_{o^{\prime} \in S^{\prime}} d\left(o^{\prime}, q\right)}{\sum_{o^{\prime} \in S_{o}} d\left(o^{\prime}, q\right)} \leq \frac{d(o, q)+w^{\prime}}{d(o, q)+w_{o}} \\
& \leq \frac{d(o, q)+H_{|\psi|} w_{o}}{d(o, q)+w_{o}} \leq H_{|\psi|}
\end{aligned}
$$

Thus, the approximation ratio is not larger than $H_{|\psi|}$, where $|\psi|<$ $|q . \psi|$, when cost $_{\text {Sum }}$ is used.

Case 1(b). $\alpha=0.5, \phi_{1}=1, \phi_{2}=1\left(\operatorname{cost}_{\text {SumMax }}\right)$.

$$
\begin{aligned}
\frac{\operatorname{cost}\left(S^{\prime} \mid 0.5,1,1\right)}{\operatorname{cost}\left(S_{o} \mid 0.5,1,1\right)} & =\frac{\sum_{o \in S^{\prime}} d(o, q)+\max _{o, o^{\prime} \in S^{\prime}} d\left(o, o^{\prime}\right)}{\sum_{o \in S_{o}} d(o, q)+\max _{o, o^{\prime} \in S_{o}} d\left(o, o^{\prime}\right)} \\
& \leq \frac{d(o, q)+w^{\prime}+d\left(o_{1}, q\right)+d\left(o_{2}, q\right)}{d(o, q)+w_{o}} \\
& \leq \frac{2\left(d(o, q)+w^{\prime}\right)}{d(o, q)+w_{o}} \\
& \leq \frac{2\left(d(o, q)+H_{|\psi|} w_{o}\right)}{d(o, q)+w_{o}} \leq 2 H_{|\psi|}
\end{aligned}
$$

where $\left(o_{1}, o_{2}\right)=\arg \max _{o, o^{\prime} \in S^{\prime}} d\left(o, o^{\prime}\right)$ and $d\left(o_{1}, o_{2}\right) \leq$ $d\left(o_{1}, q\right)+d\left(o_{2}, q\right)$ by triangle inequality.

Thus, the approximation ratio is not larger than $2 H_{|\psi|}$, where $|\psi|<|q . \psi|$, when cost $_{\text {SumMax }}$ is used.

Case 1(c). $\alpha=0.5, \phi_{1}=1, \phi_{2}=\infty\left(\right.$ cost $\left._{\text {SumMax } 2}\right)$.

As proven in Lemma 4. cost SumMax $2_{\text {is equivalent to }}$ cost $_{\text {Sum }}$. Thus, the approximation bound in this case is same as that of cost $t_{\text {Sum }}$, which is $H_{|\psi|}$.

Case 2. $\phi_{1}=\infty$. There are three parameter settings in this case. Unified-A can obtain optimal solution when $\alpha=1$ (i.e. $\operatorname{cost}_{\text {Max }}$ ). Next, we discuss the case when $\alpha=0.5$.
The proof is modified from that of [17]. Let $o_{f}$ be the object in $S^{\prime}$ that is farthest from $o, r_{1}=d\left(o_{f}, o\right)$ and $r_{2}=d(o, q)$. It could be verified that all objects in $S^{\prime}$ fall in $C\left(o, r_{1}\right) \cap C\left(q, r_{2}\right)$. Besides, it could be verified that $\max _{o \in S_{o}} d(o, q)=r_{2}$ and $\max _{o, o^{\prime} \in S_{o}} d\left(o, o^{\prime}\right) \geq r_{1}$, where $S_{o}$ is the optimal solution. Therefore, we know that $\operatorname{cost}\left(S_{o} \mid 0.5, \infty, \phi_{2}\right) \geq\left(r_{1}^{\phi_{2}}+r_{2}^{\phi_{2}}\right)^{\frac{1}{\phi_{2}}}$.

In the following, we consider two cases based on the relationship between $r_{1}$ and $r_{2}$. It could be verified that $r_{1}>\sqrt{2} r_{2}$ if the diameter of $C\left(q, r_{2}\right)$ falls in $C\left(o, r_{1}\right) \cap C\left(q, r_{2}\right)$. Otherwise we have $r_{1} \leq \sqrt{2} r_{2}$.

Case $(i): r_{1} \leq \sqrt{2} r_{2}$. We denote the intersection points between the boundaries of $C\left(o, r_{1}\right)$ and $C\left(q, r_{2}\right)$ by $a$ and $b$, as shown in Figure 3 a). It is observed that $\max _{o, o^{\prime} \in S^{\prime}} d\left(o, o^{\prime}\right) \leq$ $d(a, b)$ because all objects in $S^{\prime}$ are located in $C\left(o, r_{1}\right) \cap C\left(q, r_{2}\right)$. It could be verified that $d(a, b)=2 \sqrt{r_{1}^{2}-r_{1}^{4} / 4 r_{2}^{2}}$. Then, $\operatorname{cost}\left(S^{\prime}\right) \leq\left[r_{2}^{\phi_{2}}+\left(2 \sqrt{r_{1}^{2}-r_{1}^{4} / 4 r_{2}^{2}}\right)^{\phi_{2}}\right]^{\frac{1}{\phi_{2}}}$.Therefore,

$$
\begin{aligned}
\frac{\operatorname{cost}\left(S^{\prime} \mid 0.5, \infty, \phi_{2}\right)}{\operatorname{cost}\left(S_{o} \mid 0.5, \infty, \phi_{2}\right)} & \leq\left[\frac{r_{2}^{\phi_{2}}+\left(2 \sqrt{r_{1}^{2}-r_{1}^{4} / 4 r_{2}^{2}}\right)^{\phi_{2}}}{r_{1}^{\phi_{2}}+r_{2}^{\phi_{2}}}\right]^{\frac{1}{\phi_{2}}} \\
& \leq\left[\frac{{\frac{r_{2}}{r_{1}}}^{\phi_{2}}+\left(2 \sqrt{1-r_{1}^{2} / 4 r_{2}^{2}}\right)^{\phi_{2}}}{1+\frac{r_{2}}{r_{1}} \phi_{2}}\right]^{\frac{1}{\phi_{2}}}
\end{aligned}
$$

Let $z=\frac{r_{1}}{r_{2}}$,

$$
\begin{aligned}
\frac{\operatorname{cost}\left(S^{\prime} \mid 0.5, \infty, \phi_{2}\right)}{\operatorname{cost}\left(S_{o} \mid 0.5, \infty, \phi_{2}\right)} & \leq\left[\frac{\frac{1}{z^{\phi_{2}}}+\left(2 \sqrt{1-z^{2} / 4}\right)^{\phi_{2}}}{1+\frac{1}{z^{\phi_{3}}}}\right]^{\frac{1}{\phi_{2}}} \\
& \leq\left[\frac{1+\left(z \sqrt{4-z^{2}}\right)^{\phi_{2}}}{1+z^{\phi_{2}}}\right]^{\frac{1}{\phi_{2}}}
\end{aligned}
$$

When $\phi_{2}=1$, we define $f(z)=\frac{1+z \sqrt{4-z^{2}}}{1+z}$ on $\{z \mid z \in(0, \sqrt{2}]\}$ because $r_{1} \leq \sqrt{2} r_{2}$. It could be verified that $f(z)$ is monotonically increasing on $(0,0.875)$ and is monotonically decreasing on $(0.875, \sqrt{2}]$. Thus, $f(z) \leq f(0.875)<1.375$.

When $\phi_{2}=\infty$, we define $g(z)=\frac{\max \left\{1, z \sqrt{4-z^{2}}\right\}}{\max \{1, z\}}$ on $\{z \mid z \in(0, \sqrt{2}]\}$. It could be verified that $g(z)$ is monotonically increasing on $(0,1)$ and is monotonically decreasing on $(1, \sqrt{2}]$. Thus, $g(z) \leq g(1)<\sqrt{3}$.

Case (ii): $r_{1}>\sqrt{2} r_{2}$. Let diam $=2 r_{2}$ be the diameter of $C\left(q, r_{2}\right)$ and falls in $C\left(o, r_{1}\right) \cap C\left(q, r_{2}\right)$, as shown in Figure 3 b). Similar to case 1 , it could be verified that $\max _{o, o^{\prime} \in S^{\prime}} d\left(o, o^{\prime}\right) \leq$ diam $=2 r_{2}$. Therefore,

$$
\frac{\operatorname{cost}\left(S^{\prime} \mid 0.5, \infty, \phi_{2}\right)}{\cos \left(S_{o} \mid 0.5, \infty, \phi_{2}\right)} \leq\left[\frac{r_{2}^{\phi_{2}}+\left(2 r_{2}\right)^{\phi_{2}}}{r_{1}^{\phi_{2}}+r_{2}^{\phi_{2}}}\right]^{\frac{1}{\phi_{2}}} \leq\left[\frac{1^{\phi_{2}}+2^{\phi_{2}}}{\sqrt{2^{\phi_{2}}}+1^{\phi_{2}}}\right]^{\frac{1}{\phi_{2}}}
$$

It could be verified that $\frac{1+2}{\sqrt{2}+1} \leq 1.25$ when $\phi_{2}=1$. When $\phi_{2}=\infty$, we have $\frac{\max \{1,2\}}{\max \{\sqrt{2}, 1\}} \leq \sqrt{2}$.

Based on the above analysis, we can obtain the approximation bounds of the two sub-cases as follows. 


\begin{tabular}{|c|c|c|c|c|c|}
\hline \multirow{2}{*}{ Cost function } & \multicolumn{3}{|c|}{ Parameter } & \multirow{2}{*}{$\begin{array}{c}\text { Unified-A } \\
\text { Appro. ratio }\end{array}$} & \multirow{2}{*}{$\begin{array}{l}\text { Best known } \\
\text { Appro. ratio }\end{array}$} \\
\hline & $\alpha$ & $\phi_{1}$ & $\phi_{2}$ & & \\
\hline $\operatorname{cost}_{\Lambda}$ & 0.5 & $-\infty$ & 1 & 2 & 3 [2] \\
\hline $\operatorname{cost}_{M}$ & 0.5 & $-\infty$ & $\infty$ & 2 & N.A. \\
\hline cost & 1 & 1 & - & $H_{|\psi|}$ & $H_{\mid q .}$. \\
\hline $\operatorname{cost}_{S}$ & 0.5 & 1 & 1 & $2 H_{\mid q . \psi}$ & N.A. \\
\hline cost & 0.5 & 1 & $\infty$ & $H_{|\psi|}$ & $\left.H_{|q . \psi|} \mid 2\right]$ \\
\hline $\operatorname{cost}_{M}$ & 0.5 & $\infty$ & 1 & 1.375 & $1.375[17]$ \\
\hline $\operatorname{cost}_{N}$ & 0.5 & $\infty$ & $\infty$ & $\sqrt{3}$ & $\sqrt{3}[17]$ \\
\hline cost & 1 & $\infty$ & - & 1 & N.A. \\
\hline cost $_{\text {Min }}$ & 1 & $-\infty$ & - & 1 & N.A. \\
\hline
\end{tabular}

TABLE 5: Approx. ratios of Unified-A and existing solutions

Case 2(a). $\alpha=0.5, \phi_{1}=\infty, \phi_{2}=1\left(\operatorname{cost}_{\operatorname{MaxMax}}\right)$. The approximation ratio of the algorithm is not larger than $\max \{1.375,1.25\}=1.375$.

Case 2(b). $\alpha=0.5, \phi_{1}=\infty, \phi_{2}=\infty\left(\operatorname{cost}_{\operatorname{MaxMax2} 2}\right)$. The approximation ratio of the algorithm is not larger than $\max \{\sqrt{3}, \sqrt{2}\}=\sqrt{3}$.

Case 3. $\phi_{1}=-\infty$. There are three parameter settings in this case. Unified-A can obtain optimal solution when $\alpha=1$ (i.e. $\left.\operatorname{cost}_{\mathrm{Min}}\right)$. In the following, we discuss the case when $\alpha=0.5$. Let $o_{f}$ be the object in $S^{\prime}$ that is farthest from $o, r_{1}=d\left(o_{f}, o\right)$ and $r_{2}=d(o, q)$, as shown in Figure 3 (c). Besides, it could be verified that $\max _{o, o^{\prime} \in S_{o}} d\left(o, o^{\prime}\right) \geq r_{1}$, where $S_{o}$ is the optimal solution. Thus, we have

$$
\frac{\operatorname{cost}\left(S^{\prime} \mid 0.5,-\infty, \phi_{2}\right)}{\operatorname{cost}\left(S_{o} \mid 0.5,-\infty, \phi_{2}\right)} \leq\left[\frac{r_{2}^{\phi_{2}}+\left(2 r_{1}\right)^{\phi_{2}}}{r_{2}^{\phi_{2}}+r_{1}^{\phi_{2}}}\right]^{\frac{1}{\phi_{2}}}
$$

The approximation bounds of the two sub-cases are shown as follows.

Case 3(a). $\alpha=0.5, \phi_{1}=-\infty, \phi_{2}=1\left(\operatorname{cost}_{\text {MinMax }}\right)$.

$$
\frac{\operatorname{cost}\left(S^{\prime} \mid 0.5,-\infty, 1\right)}{\operatorname{cost}\left(S_{o} \mid 0.5,-\infty, 1\right)} \leq 2-\frac{r_{2}}{r_{2}+r_{1}} \leq 2
$$

The approximation ratio is not larger than 2 in this case.

Case 3(b). $\alpha=0.5, \phi_{1}=-\infty, \phi_{2}=\infty\left(\operatorname{cost}_{\operatorname{MinMax2} 2}\right)$.

We consider the following 3 sub-cases.

Case (i): $r_{2}>2 r_{1}$

$$
\frac{\operatorname{cost}\left(S^{\prime} \mid 0.5,-\infty, \infty\right)}{\operatorname{cost}\left(S_{o} \mid 0.5,-\infty, \infty\right)} \leq \frac{r_{2}}{r_{2}} \leq 1
$$

Case (ii): $2 r_{1} \geq r_{2}>r_{1}$

$$
\frac{\operatorname{cost}\left(S^{\prime} \mid 0.5,-\infty, \infty\right)}{\operatorname{cost}\left(S_{o} \mid 0.5,-\infty, \infty\right)} \leq \frac{2 r_{1}}{r_{2}} \leq 2
$$

Case (iii): $r_{1} \geq r_{2}$

$$
\frac{\operatorname{cost}\left(S^{\prime} \mid 0.5,-\infty, \infty\right)}{\operatorname{cost}\left(S_{o} \mid 0.5,-\infty, \infty\right)} \leq \frac{2 r_{1}}{r_{1}} \leq 2
$$

Thus, the approximation ratio is not larger than 2 .

According to the results in Table 5, we know that in despite of the fact that our unified approach is designed for a unified cost function which could be instantiated to many different cost functions, the approximate algorithm based on the unified approach

\begin{tabular}{|c|c|c|}
\hline Cost function & Exact Algorithm & Appro. Algorithm \\
\hline $\operatorname{cost}_{\text {MinMax }}$ & Cao-E1 [2] & Cao-A1 [2] \\
\hline cost $_{\text {MinMax2 }}$ & Cao-E1 [2]* & Cao-A1 [2]* \\
\hline $\operatorname{cost}_{\text {Sum }}$ & Cao-E2 [2] & Cao-A3 [2] \\
\hline $\operatorname{cost}_{\text {SumMax }}$ & Cao-E1 [2]* & Cao-A3 [2]* \\
\hline $\operatorname{cost}_{\text {MaxMax }}$ & $\begin{array}{l}\text { Cao-E1 [2], } \\
\text { Long-E } 117]\end{array}$ & $\begin{array}{l}\text { Cao-A1 } 2], \\
\text { Cao-A2 [2], } \\
\text { Long-A |17] }\end{array}$ \\
\hline $\operatorname{cost}_{\operatorname{Max} M a x 2}$ & $\begin{array}{l}\text { Cao-E1 }[2]^{*}, \\
\text { Long-E }[17]\end{array}$ & $\begin{array}{l}\text { Cao-A1 }[2]^{*}, \\
\text { Cao-A2 }[2]^{*}, \\
\text { Long-A }[17]\end{array}$ \\
\hline
\end{tabular}
provides better (same) approximation ratios than (as) the state-ofthe arts for three (two) existing cost functions.

\begin{tabular}{|c|c|c|c|}
\hline & Hotel & GN & Web \\
\hline \hline Number of objects & 20,790 & $1,868,821$ & 579,727 \\
\hline Number of unique words & 602 & 222,409 & $2,899,175$ \\
\hline Number of words & 80,645 & $18,374,228$ & $249,132,883$ \\
\hline
\end{tabular}

TABLE 6: Datasets used in the experiments
TABLE 7: Algorithms for comparison (those with the asterisk symbol are adaptations)

\section{EMPIRICAL STUDIES}

\subsection{Experimental Set-up}

Datasets. Following the existing studies [3], [17], [2], we used three real datasets in our experiments, namely Hotel, GN and Web. Dataset Hotel contains a set of hotels in the U.S. (www.allstays.com), each of which has a spatial location and a set of words that describe the hotel (e.g., restaurant, pool). Dataset GN was collected from the U.S. Board on Geographic Names (geonames.usgs.gov), where each object has a location and also a set of descriptive keywords (e.g., a geographic name such as valley). Dataset Web was generated by merging two real datasets. One is a spatial dataset called TigerCensusBlock ${ }^{2}$, which contains a set of census blocks in Iowa, Kansas, Missouri and Nebraska. The other is WEBSPAM-UK200 ${ }^{3}$, which consists of a set of web documents. Table 6 shows the statistics of the three datasets.

Query Generation. Let $O$ be a dataset of objects. Given an integer $k$, we generate a query $q$ with $k$ query keywords similarly as [3], [17] did. Specifically, to generate $q . \lambda$, we randomly pick a location from the MBR of the objects in $O$, and to generate $q . \psi$, we first rank all the keywords that are associated with objects in $O$ in descending order of their frequencies and then randomly pick $k$ keywords in the percentile range of $[10,40]$.

Cost functions. We study all instantiations of our unified cost function except for $\operatorname{cost}_{\text {Min }}$ and $\operatorname{cost}_{\text {SumMax2 }}$ since as we mentioned in Section 3, the former is of no interest and the latter is equivalent to $\operatorname{cost}_{\text {Sum }}$. That is, we study 7 cost functions in total, namely $\operatorname{cost}_{M i n M a x}, \operatorname{cost}_{\text {MinMax2 }}, \operatorname{cost}_{\text {Sum }}$ and $\operatorname{cost}_{\text {SumMax2 }}, \operatorname{cost}_{\text {MaxMax }}, \operatorname{cost}_{\text {MaxMax2 }}$ and $\operatorname{cost}_{\text {Max }}$.

Algorithms. Both the Unified-E algorithm and the Unified-A algorithm are studied. For comparison, for the CoSKQ problem with an existing cost function, the state-of-the-art algorithms are used and for the CoSKQ problem with a new cost function, some adaptions of existing algorithms are used. The state-of-theart algorithms are presented in Table 7, where Cao-E1, Cao-E2, Cao-A1, Cao-A2 and Cao-A3 refer to the algorithms MAXMAXExact, SUM-Exact, MAXMAX-Appro1, MAXMAX-Appro2 and SUM-Appro [2], respectively, and Long-E and Long-A refer to the algorithms MaxSum-Exact and MaxSum-Appro [17], respectively. Note that though the cost function $\operatorname{cost}_{\text {SumMax }}$ was proposed in [2], it was left as future work to develop solutions and thus we adapt some existing algorithms for the CoSKQ problem with this cost function.

All experiments were conducted on a Linux platform with a $2.66 \mathrm{GHz}$ machine and 32GB RAM. The IR-tree index structure is memory resident.

2. http://www.rtreeportal.org

3. http://barcelona.research.yahoo.net/webspam/datasets/uk2007 


\subsection{Experimental Results}

Following the existing studies [3], [17], [2], we used the running time and the approximation ratio (for approximate algorithms only) as measurements. Note that different sets of objects with the same costs are treated equally, and thus precision or recall are not used as measures in our experiments. For each experimental setting, we generated 500 queries and ran the algorithms with each of these queries. The average, minimum, and maximum approximation ratios were recorded and shown with bar charts.

\subsubsection{Effect of $|q . \psi|$}

Following the existing studies [3], [17], we vary the number of query keywords (i.e., $|q . \psi|$ ) from $\{3,6,9,12,15\}$. The results on the dataset Hotel are presented and those on the datasets GN and Web are similar and could be found in Appendix E

(1) $\operatorname{cost}_{\text {MinMax }}$. The results for $\operatorname{cost}_{\text {MinMax }}$ on the dataset Hotel are shown in Figure 4 According to Figure $4(a)$, the running time of each algorithm increases when $|q . \psi|$ increases. Our exact algorithm Unified-E runs consistently faster than the state-of-theart algorithm Cao-El and the gap becomes larger when $|q . \psi|$ increases. This could be explained by the fact Cao-El performs the expensive exhaustive search on the pivot objects whose number increases fast with $|q . \psi|$ while Unified-E only need to search on the regions that are possible to contain the object sets. Besides, our approximate algorithm Unified-A runs quite fast, e.g., less than 0.1 seconds, though it is slower than Cao-Al. According to Figure 4 (b), Unified-A has its approximation ratios consistently better than Cao-Al, e.g., the largest approximation ratios of Unified-A is at most 1.569 while the largest approximation ratios of $\mathrm{Cao}-\mathrm{Al}$ is at least 1.845 (and up to 2.317). Note that there could be an significant difference between a solution with 1.569 approximation ratio and that with 2.317 approximation ratio, though it does not seem to look so, e.g., in the case an optimal solution has its cost of $10 \mathrm{~km}$, a 1.569-approximate solution has a cost about $16 \mathrm{~km}$ and a 2.317 -approximate solution about $23 \mathrm{~km}$, then the difference is about $7 \mathrm{~km}(23 \mathrm{~km}-16 \mathrm{~km})$ which is more than half of the optimal cost. The reason could be that Unified-A performs an iterative process on the key query-object distance contributor which helps improve the approximation ratio while Cao-Al does not. Besides, we note that the approximation ratio of Unified-A is exactly 1 for more than $90 \%$ queries, while that of $\mathrm{Cao}-\mathrm{Al}$ is less than $70 \%$.

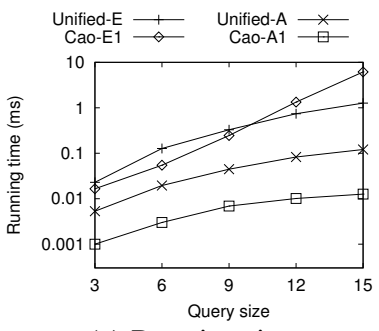

(a) Running time

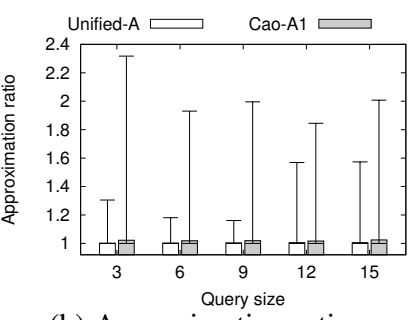

(b) Approximation ratio
Fig. 4: Effect of $|q . \psi|$ on cost $_{\text {MinMax }}$ (Hotel)

(2) $\operatorname{cost}_{\operatorname{MinMax2} 2}$. The results for $\operatorname{cost}_{\operatorname{MinMax2}}$ on the dataset Hotel are shown in Figure 5, which are similar to those for cost $_{\text {MinMax }}$, i.e., Unified-E runs consistently faster than Cao-EI and Unified-A gives better approximation ratios than $\mathrm{Cao}-\mathrm{Al}$ with reasonable efficiency.

(3) cost $_{\text {Sum }}$. The results for cost $_{\text {Sum }}$ on the dataset Hotel are shown in Figure 6 According to Figure 6 a), Unified-E runs similarly fast as $\mathrm{Cao}-E 2$ when $|q . \psi| \leq 9$ and runs faster than $\mathrm{Cao}-\mathrm{E} 2$ when $|q . \psi|>9$. Unified-E has a very restrict search space, e.g.,

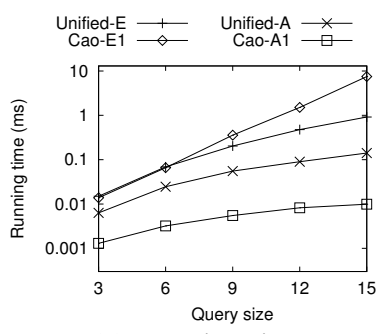

(a) Running time

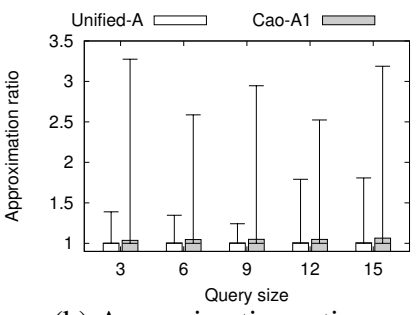

(b) Approximation ratio
Fig. 5: Effect of $|q . \psi|$ on cost $_{\text {MinMax2 }}$ (Hotel)

only those dominant objects, and Cao-E2 is a dynamic programming algorithm which might be more sensitive to $|q . \psi|$. Besides, Unified-A has a very similar running time as Cao-A3. According to Figure 6(b), Unified-A and Cao-A3 give very similar approximation ratios.

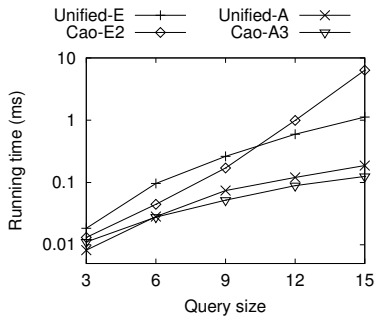

(a) Running time

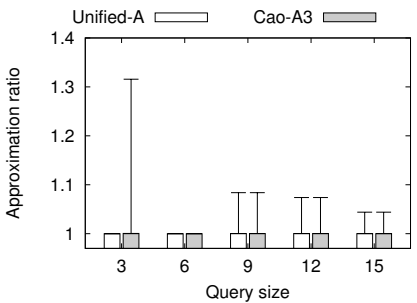

(b) Approximation ratio
Fig. 6: Effect of $|q . \psi|$ on cost $_{\text {Sum }}$ (Hotel)

(4) cost $_{\text {SumMax }}$. The results for $\operatorname{cost}_{\text {SumMax }}$ on the dataset Hotel are shown in Figure 7, which are similar to those for $\operatorname{cost}_{\text {Sum }}$ except that the competitor is $C a o-E 1$, i.e., Unified-E runs faster than $C a o-E 1$ when $|q . \psi|$ grows and Unified-A has similar running time and also approximation ratios as $\mathrm{Cao}-\mathrm{A} 3$.

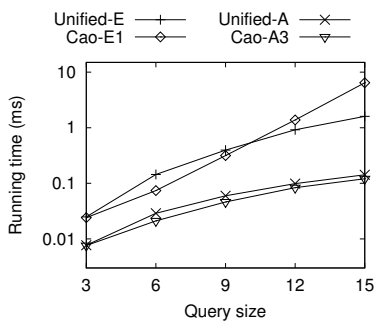

(a) Running time

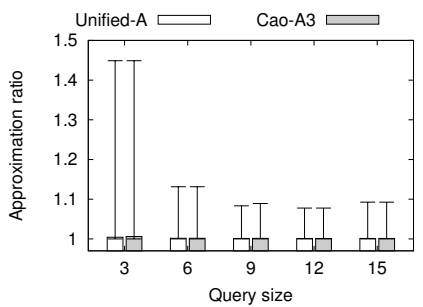

(b) Approximation ratio
Fig. 7: Effect of $|q . \psi|$ on cost $_{\text {SumMax }}$ (Hotel)

(5) $\operatorname{cost}_{\text {MaxMax }}$. The results for $\operatorname{cost}_{\text {MaxMax }}$ on the dataset Hotel are shown in Figure 8 According to Figure 8 (a), each algorithm has its running time grows when $|q . \psi|$ increases (in particular, Cao-E1 has its running time grows the fastest). Besides, Unified-E runs consistently faster than Long- $E$ and runs faster than Cao-El as well when $|q . \psi|$ gets larger. According to Figure 8 (b), all approximate algorithms including Unified-A run fast, e.g., less then 0.1 seconds, and according to Figure 8 (c), Unified-A is one of two algorithms that give the best approximation ratio (the other is Long-A). Note that Unified-A runs consistently faster than Long-A, and the reason could be that Unified-A has computation strategies based on information re-use while Long-A does not. The largest approximation ratios of Unified-A is only 1.031, while that of Cao-Al and Cao-A2 could be up to 1.904 and 1.377, respectively. Besides, Unified-A gives approximation ratio of exactly 1 for $98 \%$ queries, while that of Cao-Al and Cao-A2 are $51 \%$ and $83 \%$, respectively.

(6) $\operatorname{cost}_{\operatorname{Max} M a x 2}$. The results for $\operatorname{cost}_{\operatorname{Max} M a x 2}$ on the dataset Hotel are shown in Figure 9, which are similar as those for $\operatorname{cost}_{\text {MaxMax }}$, i.e., Unified-E has the best efficiency in general 


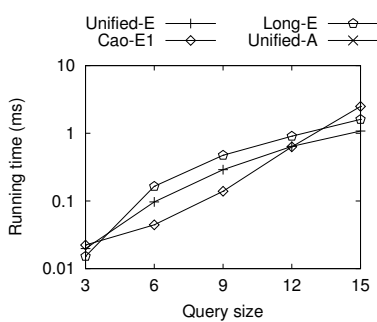

(a) Running time (Exact)

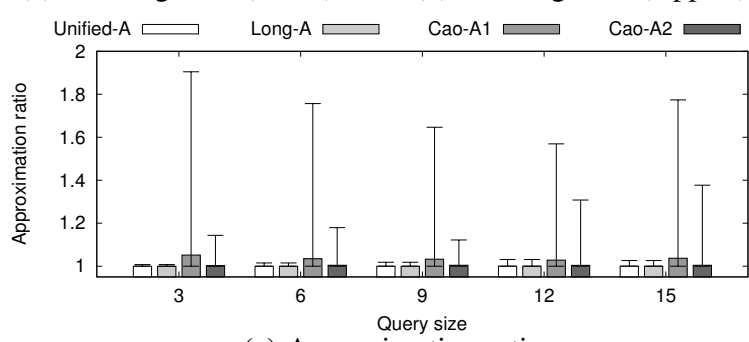

(c) Approximation ratio

Fig. 8: Effect of $|q . \psi|$ on $\operatorname{cost}_{\text {MaxMax }}$ (Hotel)

and Unified-A is among one of the two algorithms which give the best approximation ratios and also run reasonably fast. Note that the largest approximation ratios of Unified-A is only 1.080, while that of Cao-Al and Cao-A2 could be up to 1.778 and 1.347 , respectively.

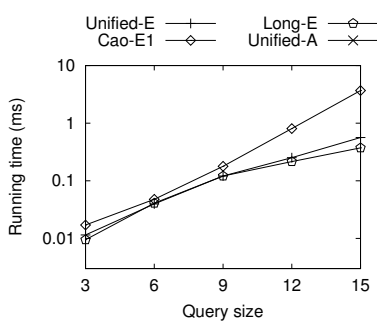

(a) Running time (Exact)

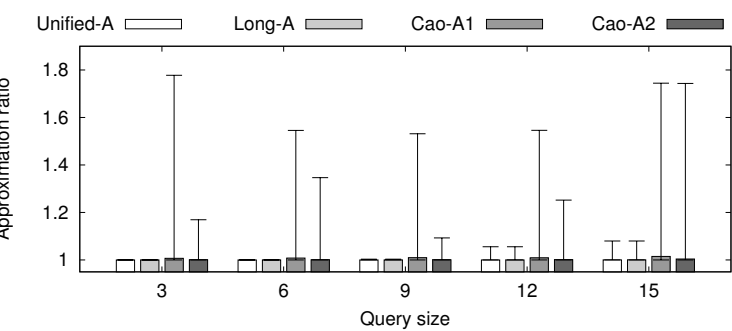

(c) Approximation ratio

Fig. 9: Effect of $|q . \psi|$ on cost $_{\text {MaxMax2 }}$ (Hotel)

(7) $\operatorname{cost}_{\text {Max }}$. The results for $\operatorname{cost}_{\text {Max }}$ are shown in Figure 16(a). According to the results, both Unified-E and Unified-A run very fast, e.g., they ran less than $0.01 \mathrm{~ms}$ for all settings of $|q . \psi|$. This is mainly because that both algorithms essentially find $N(q)$ as the solution.

\subsubsection{Effect of average $|o . \psi|$}

We further generated 5 datasets based on the Hotel dataset, where the average number of keywords an object contains (i.e. average $|o . \psi|)$ is close to $8,16,24,32$, and 40 , respectively. In the Hotel dataset, the average number of keywords an object contains is close to 4 . To generate a dataset with its average $|o . \psi|$ equal to 8 , we do the following. For each object $o$ in the Hotel dataset, we augment $o . \psi$ by including all those keywords in $o^{\prime} . \psi$ to $o . \psi$ (i.e., $o . \psi \leftarrow o . \psi \cup o^{\prime} . \psi$ ) where $o^{\prime}$ is a randomly picked object. To generate the datasets with the average $|o . \psi|$ equal to $16,24,32$ and 40 , we repeat the above process appropriate times. We vary average $|o . \psi|$ from $\{4,8,16,24,32,40\}$ and following [2], we use the default setting of $|q \cdot \psi|=10$.
(1) $\operatorname{cost}_{\text {MinMax }}$. The results for $\operatorname{cost}_{\text {MinMax }}$ are shown in Figure 10 where the results of running time of Cao-E1 for $|o . \psi| \geq 24$ are not shown simply because it ran for more than 10 hours (this applies for all the following results). According to Figure 10 a), all algorithms except for Cao-El are quite scalable when $|o . \psi|$ grows. The poor scalability of $\mathrm{CaO}-\mathrm{El}$ could be due to the fact that Cao-El is based on the search space of relevant objects around the candidate objects, which grows rapidly when $|o . \psi|$ increases. Besides, our exact algorithm Unified-E runs consistently better than Cao-E1 and Unified-A runs fast, though not as fast as Cao$A 1$, and gives obviously better approximation ratios than $\mathrm{Cao}-\mathrm{Al}$ (Figure 10 b)). Specifically, the largest approximation ratios of Unified-A is only 1.454 , which is small, while that of Cao-Al is up to 2.536 , which is not suitable for practical use.

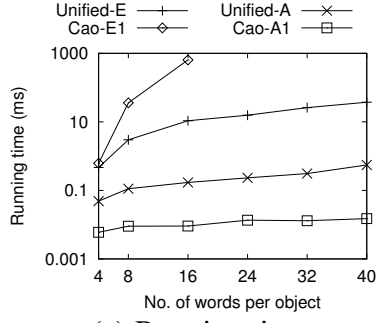

(a) Running time

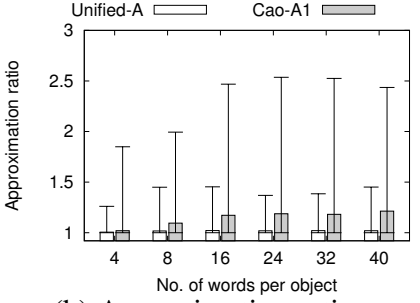

(b) Approximation ratio
Fig. 10: Effect of average $|o . \psi|$ on cost $_{\text {MinMax }}$

(2) $\operatorname{cost}_{M i n M a x 2}$. The results for cost $_{M i n M a x 2}$ are shown in Figure 11. which are similar to those for $\operatorname{cost}_{\operatorname{MinMax}}$, i.e., all algorithms except for $\mathrm{CaO}-E \mathrm{I}$ are scalable when $|o . \psi|$ grows, Unified-E runs consistently faster than Cao-E1, and Unified-A runs fast and gives the best approximation ratios.

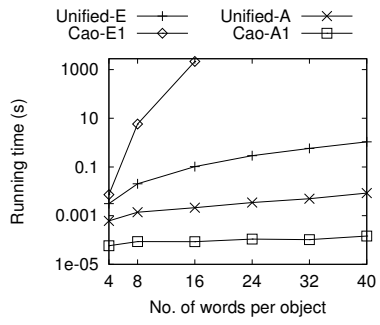

(a) Running time

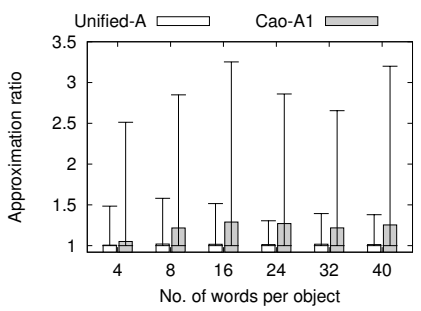

(b) Approximation ratio
Fig. 11: Effect of average $|o . \psi|$ on cost $_{\text {MinMax } 2}$ (3) cost $_{\text {Sum }}$. The results for cost $_{\text {Sum }}$ are shown in Figure 12 According to the Figure 12 a), Unified-E runs slower than Cao-E2, and the reason is perhaps that the pruning technique of Unified$E$ based on dominant objects becomes less effective when $|o . \psi|$ increases. Besides, Unified-A runs slightly slower than Cao-A3 but gives a better approximation than $\mathrm{CaO}-\mathrm{A} 3$ (Figure 12 b)). This is because Unified-A construct a feasible set for each key queryobject distance contributor and pick the best one as the solution.

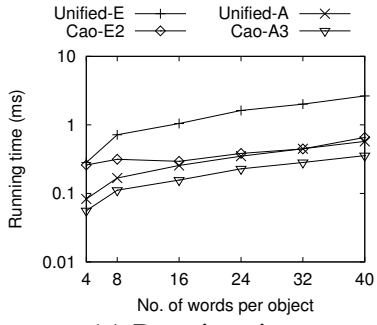

(a) Running time

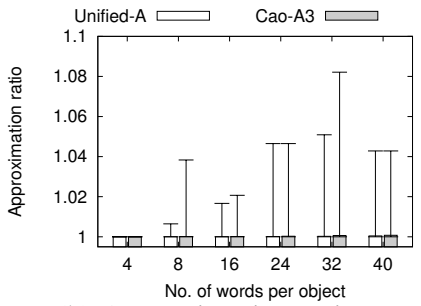

(b) Approximation ratio
Fig. 12: Effect of average $|o . \psi|$ on cost $_{\text {Sum }}$

(4) $\operatorname{cost}_{\text {SumMax }}$. Under the default setting of $|q \cdot \psi|=10$, the running times of all exact algorithms including Unified-E and CaoE1 grow very rapidly when $|o . \psi|$ increases, e.g., the algorithms 
ran for more than 1 day when $|o . \psi| \geq 8$. Thus, for better comparison among the algorithms, we particularly use the setting of $|q . \psi|=8$ for $\operatorname{cost}_{\text {SumMax }}$. According to Figure 13 (a), Unified$E$ runs consistently faster than $C a o-E 1$ and Unified-A runs fast, though not as fast as $\mathrm{Cao}-\mathrm{A} 3$, and gives a better approximation ratio (Figure 13 b)). Specifically, the largest approximation ratios of Unified-A and Cao-A3 are 1.160 and 1.251, respectively.

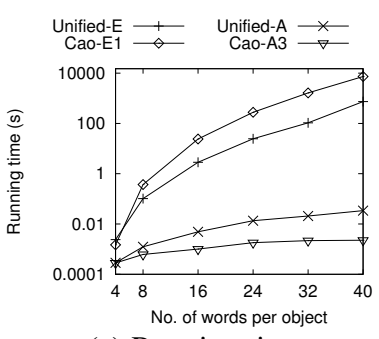

(a) Running time

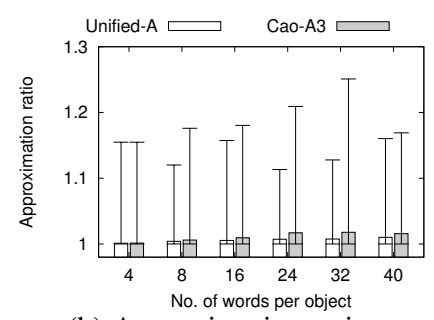

(b) Approximation ratio

Fig. 13: Effect of average $\mid$ o. $\psi \mid$ on cost $_{\text {SumMax }}$

(5) $\operatorname{cost}_{\text {MaxMax }}$. The results for $\operatorname{cost}_{\text {MaxMax }}$ are shown in Figure 14. According to Figure 14(a), Unified-E is one of the two algorithms that run the fastest and the other is Cao-E1. According to Figure 14 (b) and (c), all approximate algorithms including Unified-A run reasonably fast and Unified-A is one of the two algorithms which give the best approximation ratios (the other is Long-A). Specifically, the largest approximation ratios of Unified$A$ is only 1.135 , while that of $C a o-A 1$ and $C a o-A 2$ are 2.506 and 1.534 , respectively, which are much larger.

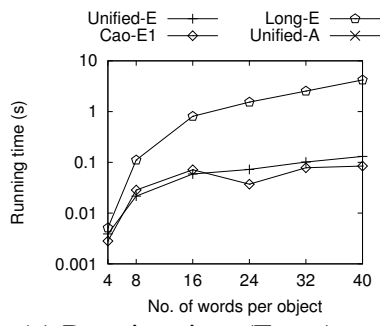

(a) Running time (Exact)

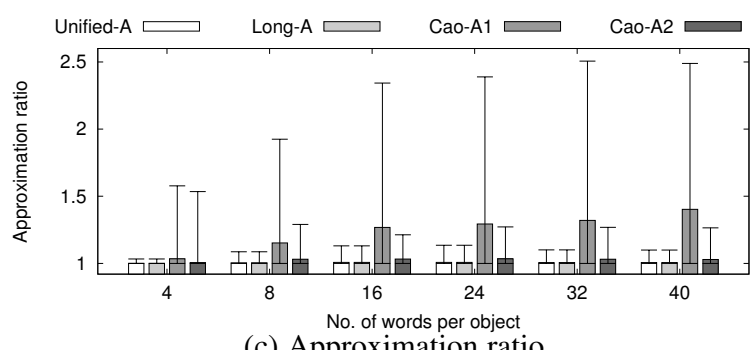

(c) Approximation ratio

Fig. 14: Effect of average $|o . \psi|$ on $\operatorname{cost}_{\text {MaxMax }}$

(6) $\operatorname{cost}_{\operatorname{MaxMax2}}$. The results for $\operatorname{cost}_{\operatorname{Max} M a x 2}$ are shown in Figure 15, which are similar to those for $\operatorname{cost}_{\text {MaxMax }}$, i.e., Unified- $E$ is one of the two fastest exact algorithm and Unified-A runs reasonably fast and is one of the two algorithms which give the best approximation ratios.

(7) cost $_{M a x}$. The results for $\operatorname{cost}_{\text {Max }}$ are shown in Figure 16(b). According to the results, both Unified-E and Unified-A run very fast, e.g., they ran less than $0.02 \mathrm{~ms}$ on all settings of $|o . \psi|$.

\subsubsection{Scalability Test}

Following the existing studies [3], [17], [2], we generated 5 synthetic datasets for the experiments of scalability test, in which the numbers of objects used are 2M, 4M, 6M, 8M and 10M. Specifically, we generated a synthetic dataset by augmenting the GN

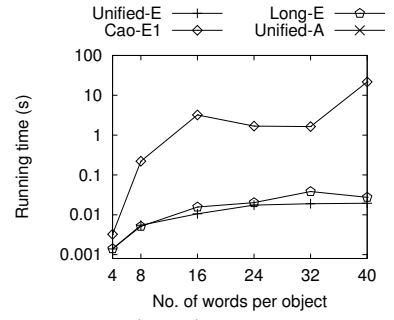

(a) Running time (Exact)

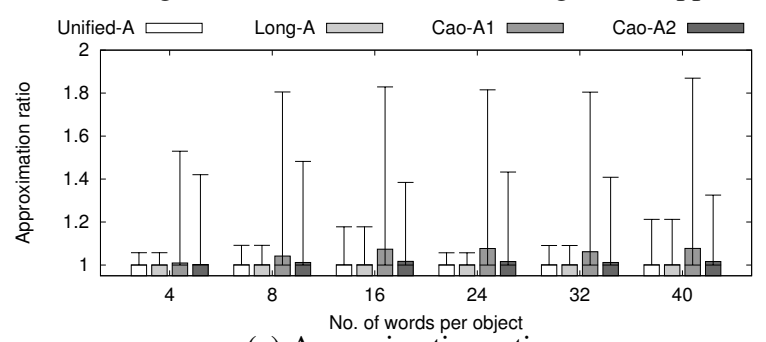

(c) Approximation ratio

Fig. 15: Effect of average $|o . \psi|$ on $\operatorname{cost}_{\text {MaxMax } 2}$

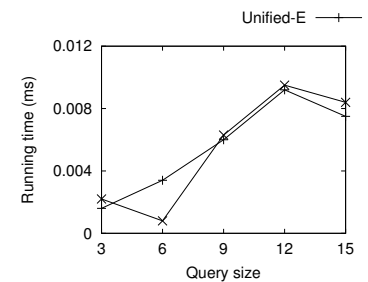

(a) Effect of $|q \cdot \psi|$

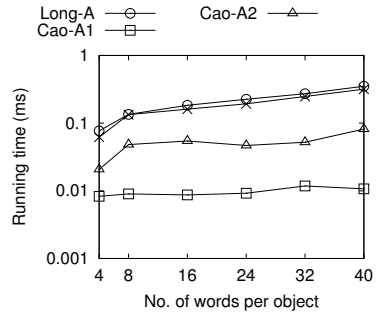

(b) Running time (Appro.)
Fig. 16: Experiments on cost $_{\operatorname{Max}}$

datasets with additional objects as follows. Each time, we create a new object $o$ with $o . \lambda$ set to be a random location from the original GN dataset by following the distribution and $o . \psi$ set to be a random document from GN and then add it into the GN dataset. We vary the number of objects from $\{2 M, 4 M, 6 M, 8 M, 10 M\}$, following [2], we use the default setting of $|q . \psi|=10$.

(1) $\operatorname{cost}_{\text {MinMax }}$. The results for $\operatorname{cost}_{\text {MinMax }}$ are shown in Figure 17. According to Figure 17,a), our exact algorithm Unified$E$ runs consistently faster than Cao-El and it is scalable wrt the number of objects, e.g., it ran within 30 seconds on a dataset with $10 \mathrm{M}$ objects. Besides, our approximate algorithm Unified-A is also scalable, e.g., it ran within 1 second on a dataset with $10 \mathrm{M}$ objects, and gives near-to-optimal approximation ratios (Figure 17(b)). The largest approximation ratios of Unified-A is only 1.622, which is very small, while that of Cao-Al is 2.692, which is not practical. This also conform with our theoretical analysis that Unified-A has a better approximation ratio than Cao-Al in $\operatorname{cost}_{\text {MinMax }}$.

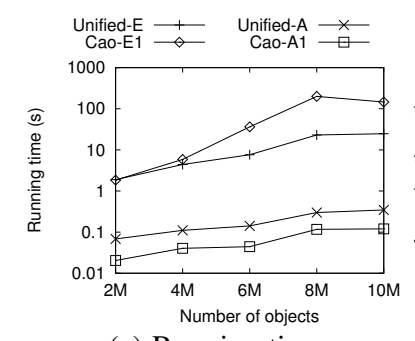

(a) Running time

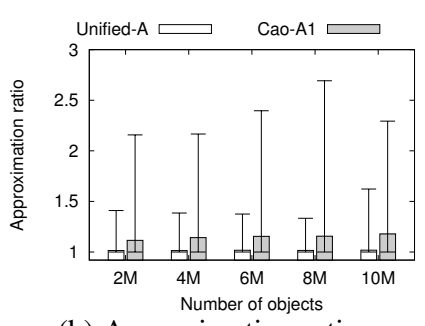

(b) Approximation ratio
Fig. 17: Scalability test on cost $_{\text {MinMax }}$

The results for the remaining cost functions are put in Appendix Fdue to the page limit. According to the results, we know that both Unified-E and Unified-A are scalable to large datasets. 


\subsection{Summary Of Experimental Results}

Our exact algorithm Unified-E is clearly the best exact algorithm for CoSKQ queries not only because it is a unified approach but also it is always among those with the best running times (e.g., it beats the state-of-the arts consistently for $\operatorname{cost}_{\text {MinMax }}$ and cost $_{\text {MinMax } 2}$, when $|q . \psi|$ becomes large for cost $_{S u m}$ and cost $_{\text {SumMax }}$, and under the majority of settings for $\operatorname{cost}_{\text {Max Max }}$ and $\operatorname{cost}_{\operatorname{Max} \operatorname{Max} 2}$ ).

Our approximate algorithm Unified-A runs reasonably fast (e.g., for the majority settings of $|q . \psi|$, it ran within 0.1 seconds), while sometimes it is not as fast as the competitors because Unified-A has some more checking so that it can take care all cost functions. Meanwhile, Unified-A is always among the those which give the best approximation ratios close to 1 and runs always faster than those algorithms which give similar approximation ratios as Unified-A.

\section{CONCLUSION}

In this paper, we proposed a unified cost function for CoSKQ. This cost function expresses all existing cost functions in the literature and a few cost functions that have not been studied before. We designed a unified approach, which consists of one exact algorithm and one approximate algorithm. The exact algorithm runs comparably fast as the existing exact algorithms, while the approximate algorithm provides a comparable approximation ratio as the existing approximate algorithms. Extensive experiments were conducted which verified our theoretical findings.

There are several interesting future research directions. One direction is to design a cost function such that it penalizes those objects with too much keywords for fairness. Another direction is to extend CoSKQ with the unified cost function to other distance metrics such as road networks. It is also interesting to extend the unified approach to handle the route-oriented spatial keyword queries. Besides, it is left as a remaining issue to study the CoSKQ problem with a moving query point.

\section{ACKNOWLEDGMENTS}

We are grateful to the anonymous reviewers for their constructive comments on this paper. The research of Harry Kai-Ho Chan and Raymond Chi-Wing Wong is supported by HKRGC GRF 16219816.

\section{REFERENCES}

[1] X. Cao, L. Chen, G. Cong, and X. Xiao. Keyword-aware optimal route search. PVLDB, 5(11):1136-1147, 2012.

[2] X. Cao, G. Cong, T. Guo, C. S. Jensen, and B. C. Ooi. Efficient processing of spatial group keyword queries. TODS, 40(2):13, 2015.

[3] X. Cao, G. Cong, C. S. Jensen, and B. C. Ooi. Collective spatial keyword querying. In SIGMOD, pages 373-384. ACM, 2011.

[4] X. Cao, G. Cong, C. S. Jensen, and M. L. Yiu. Retrieving regions of intersect for user exploration. PVLDB, 7(9), 2014.

[5] A. Cary, O. Wolfson, and N. Rishe. Efficient and scalable method for processing top-k spatial boolean queries. In SSDBM, pages 87-95. Springer, 2010.

[6] H. K.-H. Chan, C. Long, and R. C.-W. Wong. Inherent-cost aware collective spatial keyword queries. In SSTD, 2017.

[7] D.-W. Choi, J. Pei, and X. Lin. Finding the minimum spatial keyword cover. In ICDE, pages 685-696. IEEE, 2016.

[8] G. Cong, C. S. Jensen, and D. Wu. Efficient retrieval of the top-k most relevant spatial web objects. PVLDB, 2(1):337-348, 2009.

[9] G. Cong, H. Lu, B. C. Ooi, D. Zhang, and M. Zhang. Efficient spatial keyword search in trajectory databases. Arxiv preprint arXiv:1205.2880, 2012.

[10] K. Deng, X. Li, J. Lu, and X. Zhou. Best keyword cover search. TKDE, 27(1):61-73, 2015

[11] J. Fan, G. Li, L. Z. S. Chen, and J. Hu. Seal: Spatio-textual similarity search. PVLDB, 5(9):824-835, 2012.
[12] I. D. Felipe, V. Hristidis, and N. Rishe. Keyword search on spatial databases. In ICDE, pages 656-665. IEEE, 2008.

[13] Y. Gao, J. Zhao, B. Zheng, and G. Chen. Efficient collective spatial keyword query processing on road networks. ITS, 17(2):469-480, 2016.

[14] T. Guo, X. Cao, and G. Cong. Efficient algorithms for answering the $\mathrm{m}$ closest keywords query. In SIGMOD. ACM, 2015.

[15] Z. Li, K. Lee, B. Zheng, W. Lee, D. Lee, and X. Wang. Ir-tree: An efficient index for geographic document search. TKDE, 23(4):585-599, 2011.

[16] J. Liu, K. Deng, H. Sun, Y. Ge, X. Zhou, and C. Jensen. Clue-based spatio-textual query. PVLDB, 10(5):529-540, 2017.

[17] C. Long, R. C.-W. Wong, K. Wang, and A. W.-C. Fu. Collective spatial keyword queries:a distance owner-driven approach. In SIGMOD, pages 689-700. ACM, 2013.

[18] J. Rocha, O. Gkorgkas, S. Jonassen, and K. Nørvåg. Efficient processing of top-k spatial keyword queries. In SSTD, pages 205-222. Springer, 2011.

[19] J. B. Rocha-Junior and K. Nørvåg. Top-k spatial keyword queries on road networks. EDBT, pages 168-179. ACM, 2012.

[20] S. Shang, R. Ding, B. Yuan, K. Xie, K. Zheng, and P. Kalnis. User oriented trajectory search for trip recommendation. In EDBT, pages 156167. ACM, 2012.

[21] A. Skovsgaard and C. S. Jensen. Finding top-k relevant groups of spatia web objects. VLDBJ, 24(4):537-555, 2015.

[22] S. Su, S. Zhao, X. Cheng, R. Bi, X. Cao, and J. Wang. Group-based collective keyword querying in road networks. Information Processing Letters, 118:83-90, 2017.

[23] D. Wu, G. Cong, and C. Jensen. A framework for efficient spatial web object retrieval. VLDBJ, 21(6):797-822, 2012.

[24] D. Wu, M. Yiu, G. Cong, and C. Jensen. Joint top-k spatial keyword query processing. TKDE, 24(10):1889-1903, 2012.

[25] D. Wu, M. L. Yiu, C. S. Jensen, and G. Cong. Efficient continuously moving top-k spatial keyword query processing. In ICDE, pages 541552. IEEE, 2011

[26] Y. Zeng, X. Chen, X. Cao, S. Qin, M. Cavazza, and Y. Xiang. Optimal route search with the coverage of users' preferences. In IJCAI, pages 2118-2124, 2015

[27] C. Zhang, Y. Zhang, W. Zhang, and X. Lin. Inverted linear quadtree: Efficient top k spatial keyword search. In ICDE, pages 901-912. IEEE, 2013.

[28] D. Zhang, Y. M. Chee, A. Mondal, A. Tung, and M. Kitsuregawa. Keyword search in spatial databases: Towards searching by document. In ICDE, pages 688-699. IEEE, 2009.

[29] D. Zhang, B. C. Ooi, and A. K. H. Tung. Locating mapped resources in web 2.0. In ICDE, pages 521-532. IEEE, 2010.

[30] D. Zhang, K.-L. Tan, and A. K. H. Tung. Scalable top-k spatial keyword search. EDBT/ICDT, pages 359-370. ACM, 2013.
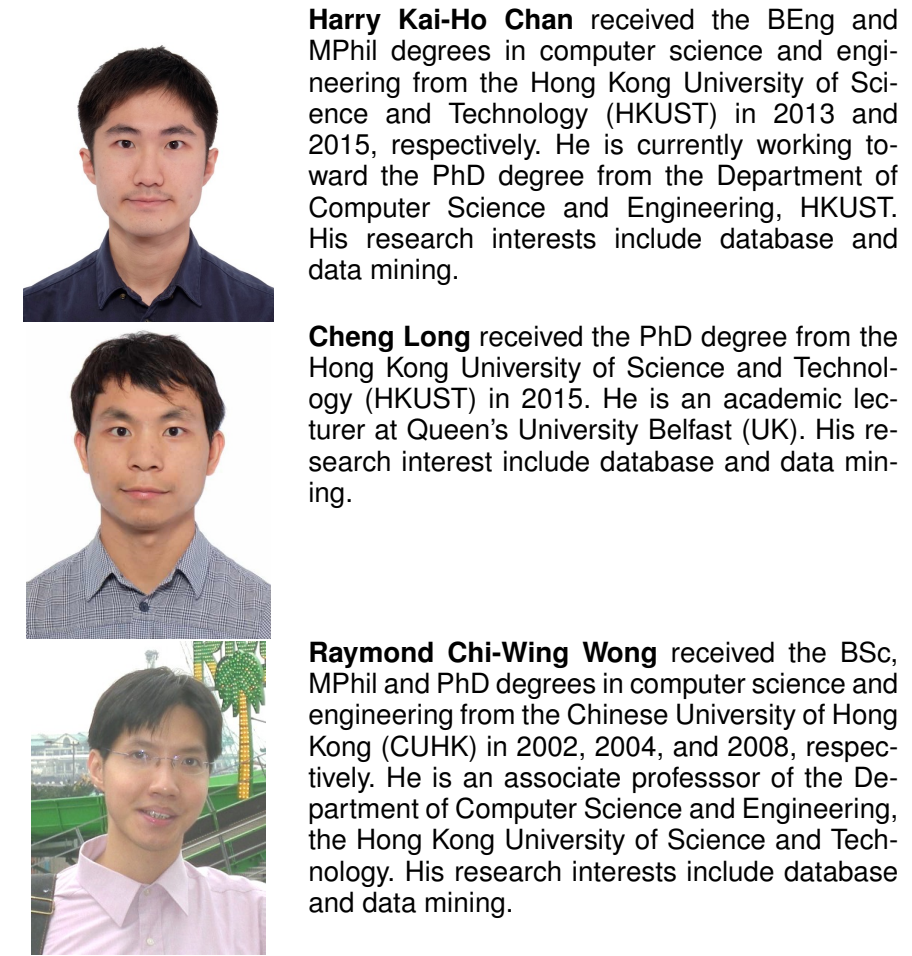

Cheng Long received the $\mathrm{PhD}$ degree from the Hong Kong University of Science and Technology (HKUST) in 2015. He is an academic lecturer at Queen's University Belfast (UK). His research interest include database and data mining.

Raymond Chi-Wing Wong received the BSc, MPhil and PhD degrees in computer science and engineering from the Chinese University of Hong Kong (CUHK) in 2002, 2004, and 2008, respectively. $\mathrm{He}$ is an associate professsor of the Department of Computer Science and Engineering the Hong Kong University of Science and Technology. His research interests include database and data mining. 


\section{APPENDIX A}

\section{EQUIVALENCE OF cost $_{\text {SumMax } 2}$ AND cost $_{\text {Sum }}$}

We have the following lemma to show the functionality of cost $_{\text {SumMax } 2}$ and cost $_{\text {Sum }}$ are equivalent.

Lemma 4. Let $S$ be an object set. $\operatorname{cost}_{\operatorname{SumMax} 2}(S)=$ cost $_{\text {Sum }}(S)$.

Proof. Let $\left(o_{1}, o_{2}\right)=\arg \max _{o_{1}, o_{2} \in S} d\left(o_{1}, o_{2}\right)$. We have

$\operatorname{cost}_{\operatorname{SumMax} 2}(S)=\max \left\{\sum_{o \in S} d(o, q), d\left(o_{1}, o_{2}\right)\right\}$. Note that $d\left(o_{1}, q\right)+d\left(o_{2}, q\right) \geq d\left(o_{1}, o_{2}\right)$ by triangle inequality and $\sum_{o \in S} d(o, q) \geq d\left(o_{1}, q\right)+d\left(o_{2}, q\right)$. Thus, $\operatorname{cost}_{\text {SumMax } 2}(S)=$ $\sum_{o \in S} d(o, q)=\operatorname{cost}_{S u m}(S)$.

This lemma suggests that it is sufficient to consider one of these two cost functions. In this paper, we focus the discussion on cost $_{\text {Sum }}$.

\section{APPENDIX B ProOF OF THEOREM 1}

We first give the decision problem of CoSKQ. Given a set $O$ of spatial objects each $o \in O$ associated with a location $o . \lambda$ and a set of keywords $o . \psi$, a query $q$ consisting of a query location $q . \lambda$ and a set of query keywords $q . \psi$, and a real number $C$, the problem is to determine whether there exists a set $S$ of objects in $O$ such that $S$ covers the query keywords and $\operatorname{cost}_{\text {unified }}(S)$ is at most $C$.

We then prove by transforming the 3-satisfiability (3-SAT) problem which is known to be NP-Complete to the CoSKQ problem and showing the equivalence between two problems. The description of the 3-SAT problem is given as follows. Let $U$ be a set of literals $\left\{e_{1}, \overline{e_{1}}, \ldots, e_{n}, \overline{e_{n}}\right\}$ where $\overline{e_{i}}$ is the negation of $e_{i}$. Given an expression $E=C_{1} \wedge C_{2} \wedge \ldots \wedge C_{m}$ where $C_{j}=x_{j} \vee y_{j} \vee z_{j}$ and $x_{j}, y_{j}, z_{j} \in U$ for $1 \leq j \leq m$, the problem is to determine whether there exists a truth assignment for $e_{i}$ for $i \leq i \leq n$ such that $E$ is true.

Based on the value of parameter $\phi_{1}$, we use different transformations.

Case 1. $\phi_{1}=1$. We construct a set $O$ of $2 n$ objects as follows. For each literal $e_{i}$ in $U$, we create an object $o_{i}$ in $O$, and for each literal $\overline{e_{i}}$ in $U$, we create an object $o_{i}^{\prime}$ in $O$. In total, there are $2 n$ objects in $O$. We set the locations of the objects in $O$ such that they are all located at the same place i.e., for any $o \in O, o . \lambda$ is identical. Besides, for each object $o_{i}(1 \leq i \leq n)$, we set $o_{i} . \psi$ such that $o_{i} . \psi$ includes a keyword $k_{i}$ corresponding to $e_{i}$ and a keyword $k_{j}^{\prime}$ corresponding to $C_{j}$ if $C_{j}$ involves $e_{i}$ for $1 \leq j \leq m$. Similarly, for each object $o_{i}^{\prime}(1 \leq i \leq n)$, we set $o_{i}^{\prime} \cdot \psi$ such that $o_{i}^{\prime}$. $\psi$ includes the keyword $k_{i}^{\prime}$ and all $k_{j}^{\prime}$ 's with $C_{j}$ involving $\overline{e_{i}}$ for $1 \leq j \leq m$. We construct a query $q$ by setting $q . \lambda$ to be a location such that $d(o, q)=1$ for any object $o \in O$ and $q \cdot \psi$ to be a set of $m+n$ keywords, $\left\{k_{1}, k_{2}, \ldots, k_{n}, k_{1}^{\prime}, k_{2}^{\prime}, \ldots, k_{m}^{\prime}\right\}$. We set $C$ to be $n$. The above transformation process could be done in polynomial time.

Case 2. $\phi_{1} \in\{\infty,-\infty\}$. We construct a set $O$ of $2 n$ objects as follows. For each literal $e_{i}$ in $U$, we create an object $o_{i}$ in $O$, and for each literal $\overline{e_{i}}$ in $U$, we create an object $o_{i}^{\prime}$ in $O$. In total, there are $2 n$ objects in $O$. For the locations of the objects, consider a circle Cir with its center at $q . \lambda$ (which is selected arbitrarily) and its radius equal to 1 . We set the locations of the objects in $O$ such that they are all located on the boundary of $\mathrm{Cir}$ such that $d\left(o_{i}, o_{i}^{\prime}\right)=2$. Besides, for each object $o_{i}(1 \leq i \leq n)$, we set $o_{i} . \psi$ such that $o_{i} . \psi$ includes a keyword $k_{i}$ corresponding to $e_{i}$ and a keyword $k_{j}^{\prime}$ corresponding to $C_{j}$ if $C_{j}$ involves $e_{i}$ for $1 \leq j \leq m$. Similarly, for each object $o_{i}^{\prime}(1 \leq i \leq n)$, we set $o_{i}^{\prime} \cdot \psi$ such that $o_{i}^{\prime} \cdot \psi$ includes the keyword $k_{i}^{\prime}$ and all $k_{j}^{\prime}$ 's with $C_{j}$ involving $\overline{e_{i}}$ for $1 \leq j \leq m$. We construct a query $q$ by setting $q . \lambda$ arbitrarily and $q . \psi$ to be a set of $m+n$ keywords, $\left\{k_{1}, k_{2}, \ldots, k_{n}, k_{1}^{\prime}, k_{2}^{\prime}, \ldots, k_{m}^{\prime}\right\}$. The above transformation process could be done in polynomial time. We consider the following subcases for setting $C$.

Case 2(a). $\phi_{2}=1$. We set $C=3-\epsilon$ where $\epsilon$ is close to zero.

Case 2(b). $\phi_{2}=\infty$. We set $C=2-\epsilon$ where $\epsilon$ is close to zero.

We show the equivalence between two problem instances as follows. Suppose that the answer of the 3-SAT problem is "yes", i.e., there exists a truth assignment for the literals in $U$ such that $E$ is correct. We denote the truth assignment by a set $T$ of literals which are true under the assignment. Note that $T$ has exactly $n$ literals and $e_{i}$ and $\overline{e_{i}}$ do not appear in $T$ simultaneously for any $1 \leq i \leq n$. Then, it could be verified that the set of objects each corresponding to a literal in $T$ covers $q . \psi$ and the cost of the set at most $C$, and thus the answer of the CoSKQ problem is also "yes". Suppose that the answer of the CoSKQ problem is "yes". Let $S$ be the set of objects in $O$ that covers $q . \psi$ and has the cost at most $C$. We know that object $o_{i}$ and $o_{i}^{\prime}$ are not included in $S$ simultaneously. It could be verified that with the truth assignment represented by the set of literals corresponding to the objects in $S, E$ is correct, and thus the answer of the 3-SAT problem is also "yes".

\section{ApPENDIX C Pruning based on DOMINANCE}

To improve the efficiency of the algorithm, we propose a pruning strategy to prune the search space when $\alpha=1$ and $\phi_{1}=1$. Before we give the strategy, we first introduce the concept of dominance. Given a query $q$, two objects $o_{1}$ and $o_{2}$, we say $o_{1}$ dominate $o_{2}$ if the following two conditions are satisfied. (1) $d\left(o_{1}, q\right)<d\left(o_{2}, q\right)$, and (2) all keywords in $q . \psi$ that are covered by $O_{2}$ can be covered by $o_{1}$, (i.e. $q . \psi \cap o_{1} \cdot \psi \supseteq q . \psi \cap o_{2} \cdot \psi$ ). A dominant object is defined to be an object that is not dominated by any other objects. Then we have the following lemma to prune the objects that are not dominant objects.

Lemma 5. When $\alpha=1$ and $\phi_{1}=1$, all objects in the optimal solution $S$ are dominant objects.

Proof: We prove this by contradiction. Let an object $o \in S$ that is not a dominant object. Then, there must exist an object $o^{\prime}$ that dominate $o$. Note that $o^{\prime}$ also covers the query keywords covered by $o$ and is closer to $q$. We can construct a better solution $S^{\prime}=$ $S \backslash\{o\} \cup\left\{o^{\prime}\right\}$, which contradicts the fact that $S$ is the optimal solution.

Based on this lemma, it is sufficient for the algorithm to consider the dominant objects only when enumerating the object sets. Specifically, whenever the algorithm performs a range query, it discards the objects that are being dominated and proceeds with the dominant objects.

\section{APPENDIX D \\ BETTER IMPLEMENTATION BASED ON INFORMATION RE-USE}

To implement the Unified-A algorithm efficiently, we have the following computation strategies. First, when the algorithm finding 


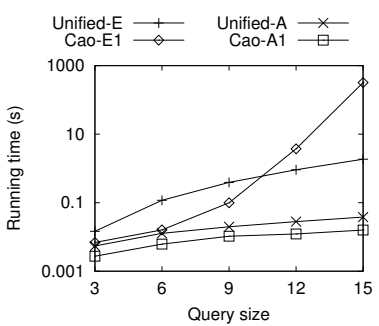

(a) Running time

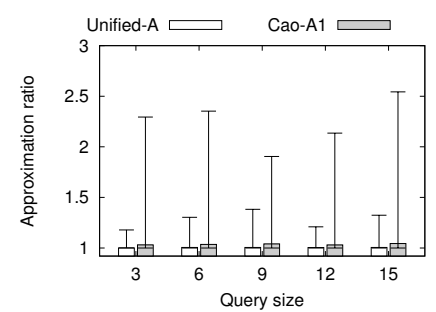

(b) Approximation ratio
Fig. 18: Effect of $|q . \psi|$ on cost $_{\text {MinMax }}(\mathrm{GN})$

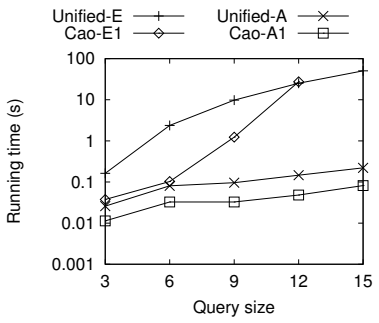

(a) Running time

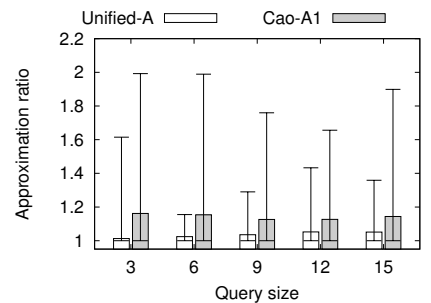

(b) Approximation ratio
Fig. 19: Effect of $|q . \psi|$ on $\operatorname{cost}_{\text {MinMax }}$ (Web)

the set of all relevant objects in $R_{o}$ (line 5 in Algorithm 4), instead of issuing a range query in each iteration, it re-uses the information from the previous iteration by maintaining the region $R_{o}$ dynamically. Specifically, consider one iteration. The algorithm finds a feasible set that has an object $o$ as a key query-object distance contributor in the region $R_{o}$. After it finishes the current iteration, it adds $o$ into $R_{o}$ (when $\phi_{1} \in\{1, \infty\}$ ), or removes $o$ from $R_{o}$ (when $\phi_{1}=-\infty$ ).

Second, when the algorithm performs the iterative process (lines 8-14 in Algorithm 4), instead of searching for the object with minimum ratio (distance) from $\mathcal{O}^{\prime}$ in each iteration, it maintains a heap structure for storing the objects. Specifically, when $\phi_{1}=1$, the key of the objects in the heap are the ratios, and the heap is updated after each object is picked. When $\phi_{1} \in\{\infty,-\infty\}$, the key of the objects in the heap are the distances, and in each iteration the algorithm picks the relevant object with the smallest distance.

\section{APPENDIX E}

\section{EXPERIMENTAL RESUlts on the DATASETS GN AND WEB}

In the following, we present the experimental results on the datasets GN and Web of varying $|q . \psi|$. Following the existing studies [3], [17], we vary the number of query keywords (i.e., $|q . \psi|)$ from $\{3,6,9,12,15\}$.

(1) $\operatorname{cost}_{\text {MinMax }}$. The results for $\operatorname{cost}_{\text {MinMax }}$ on the datasets GN and Web are shown in Figure 18 and Figure 19 , respectively, which are similar to that on the dataset Hotel. The result of running time of Cao-E1 for $|q . \psi|=15$ is not shown in Figure 19 simply because it ran for more than 10 hours (this applies for all the following results).

(2) $\operatorname{cost}_{\operatorname{MinMax} 2}$. The results for $\operatorname{cost}_{\operatorname{MinMax} 2}$ on the datasets GN and Web are shown in Figure 20 and Figure 21, respectively, which are similar to those for cost MinMax.

(3) cost $_{\text {Sum }}$. The results for cost $_{\text {Sum }}$ on the datasets GN and Web are shown in Figure 22 and Figure 23, respectively. According to the results, Unified-E runs slower than Cao-E2 but still within a reasonable time (e.g. within 10 seconds on the largest dataset

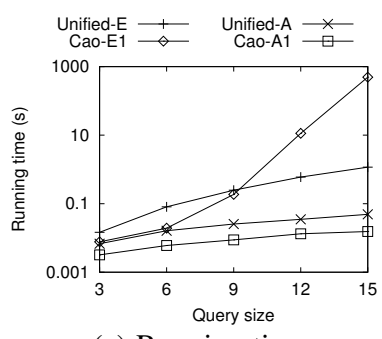

(a) Running time

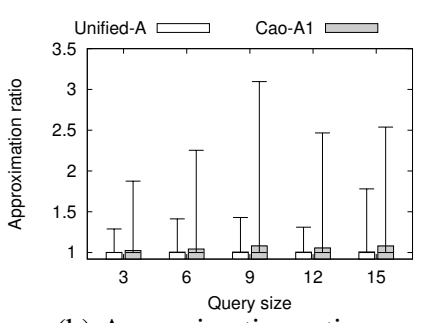

(b) Approximation ratio
Fig. 20: Effect of $|q . \psi|$ on $\operatorname{cost}_{\operatorname{MinMax2}}(\mathrm{GN})$

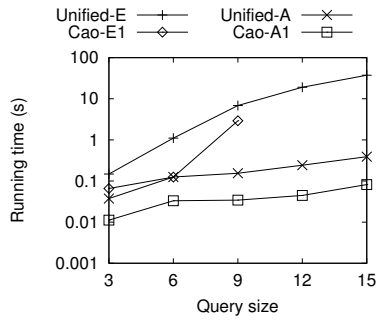

(a) Running time

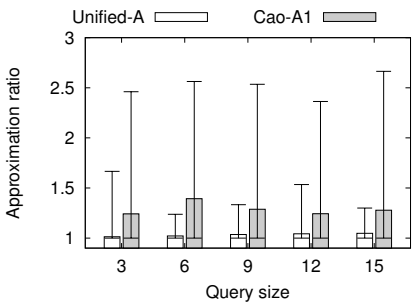

(b) Approximation ratio
Fig. 21: Effect of $|q . \psi|$ on $\operatorname{cost}_{\operatorname{MinMax} 2}$ (Web)

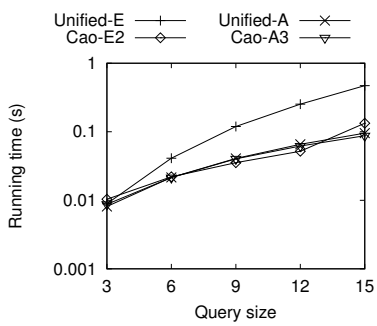

(a) Running time

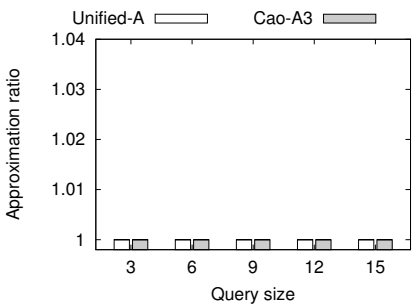

(b) Approximation ratio
Fig. 22: Effect of $|q . \psi|$ on cost $_{\text {Sum }}$ (GN)

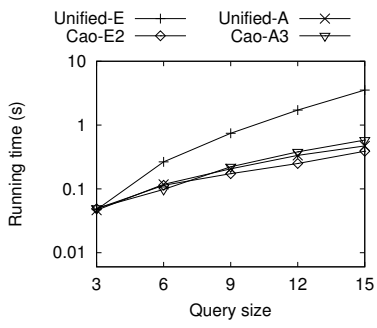

(a) Running time

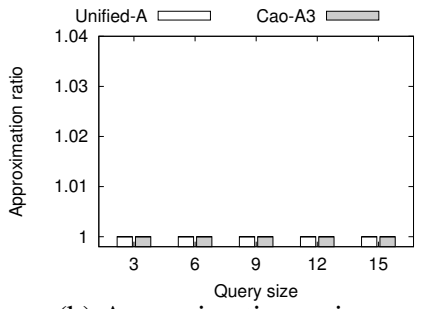

(b) Approximation ratio
Fig. 23: Effect of $|q . \psi|$ on cost $_{\text {Sum }}$ (Web)

Web). Besides, Unified-A has a very similar running time as $\mathrm{Cao}$ $A 3$, while Unified-A can always obtain an approximation ratios of 1.

(4) $\operatorname{cost}_{\text {SumMax }}$. The results for cost $_{\text {SumMax }}$ on the datasets GN and Web are shown in Figure 24 and Figure 25, respectively, which are similar to that on the dataset Hotel.

(5) $\operatorname{cost}_{\text {MaxMax }}$. The results for $\operatorname{cost}_{\text {MaxMax }}$ on the datasets GN and Web are shown in Figure 26 and Figure 27, respectively, which are similar to that on the dataset Hotel.

(6) $\operatorname{cost}_{\operatorname{Max} M a x 2}$. The results for $\operatorname{cost}_{\operatorname{Max} M a x 2}$ on the datasets GN and Web are shown in Figure 28 and Figure 29. respectively, which are similar to that on the dataset Hotel.

(7) $\operatorname{cost}_{M a x}$. The results for $\operatorname{cost}_{\text {Max }}$ on the datasets GN and Web are shown in Figure 30, which is similar to that on the dataset Hotel. According to the results, both Unified-E and Unified-A run very fast, e.g. they ran less than $6 \mathrm{~ms}$ for all settings of $|q . \psi|$. 


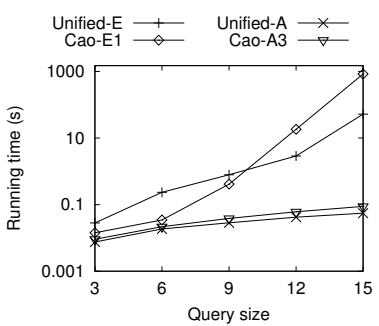

(a) Running time

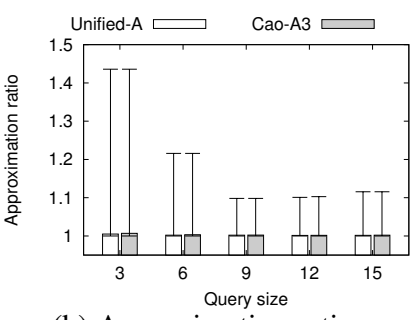

(b) Approximation ratio
Fig. 24: Effect of $|q . \psi|$ on cost $_{\text {SumMax }}(\mathrm{GN})$

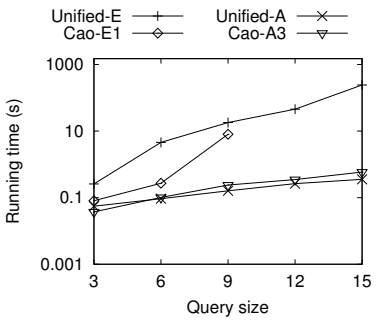

(a) Running time

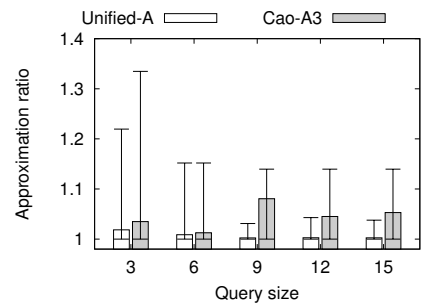

(b) Approximation ratio
Fig. 25: Effect of $|q . \psi|$ on cost $_{\text {SumMax }}$ (Web)

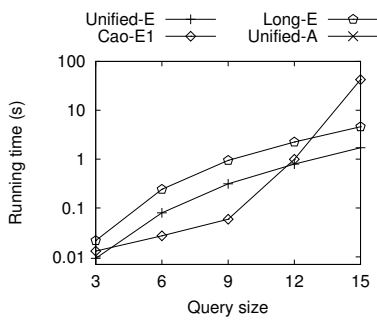

(a) Running time (Exact)
Long-A $\square$
Cao-A1 $\square$

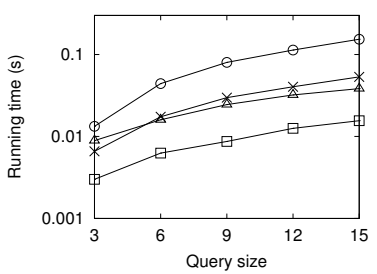

(b) Running time (Appro.)

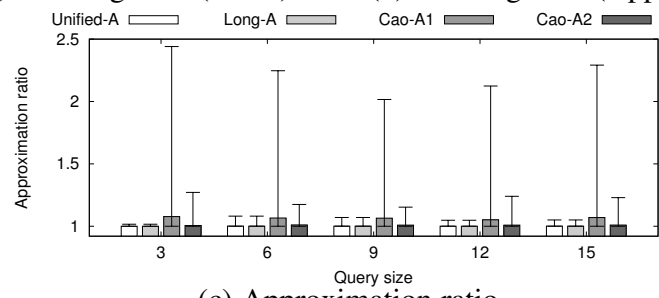

(c) Approximation ratio

Fig. 26: Effect of $|q . \psi|$ on $\operatorname{cost}_{\text {Max Max }}(\mathrm{GN})$

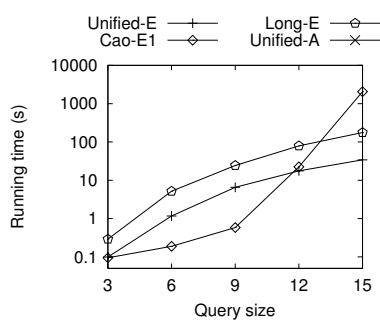

(a) Running time (Exact)

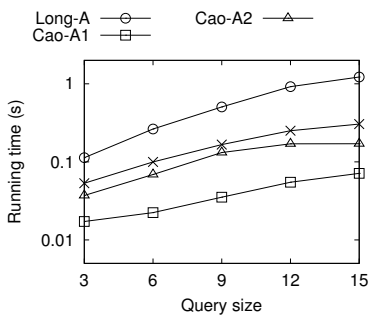

(b) Running time (Appro.)

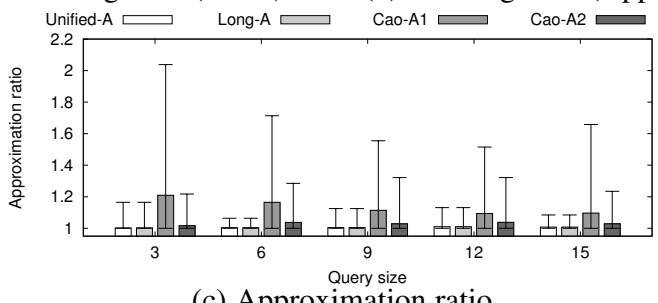

(c) Approximation ratio

Fig. 27: Effect of $|q . \psi|$ on cost $_{\text {MaxMax }}$ (Web)

\section{APPENDIX $F$}

\section{SCALABILITY TEST}

(2) $\operatorname{cost}_{\operatorname{MinMax2}}$. The results for $\operatorname{cost}_{\text {MinMax } 2}$ are shown in Figure 31 According to Figure 31.a), Unified-E is faster and more

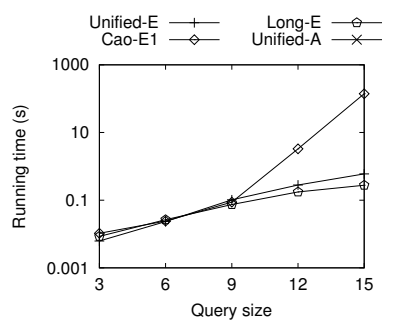

(a) Running time (Exact)
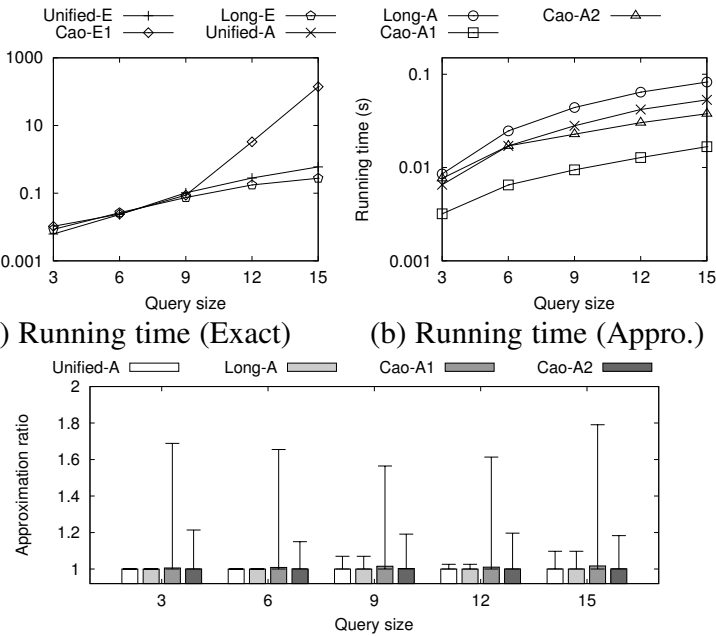

(c) Approximation ratio

Fig. 28: Effect of $|q . \psi|$ on $\operatorname{cost}_{\operatorname{Max} \operatorname{Max} 2}(\mathrm{GN})$

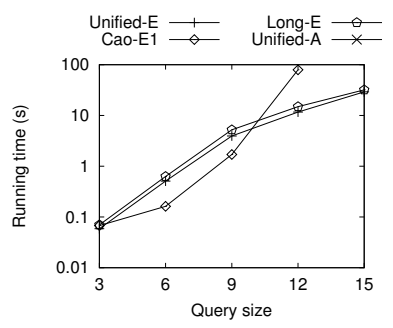

(a) Running time (Exact)

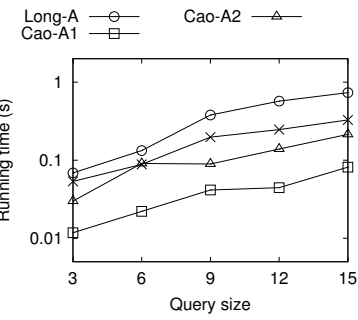

(b) Running time (Appro.)

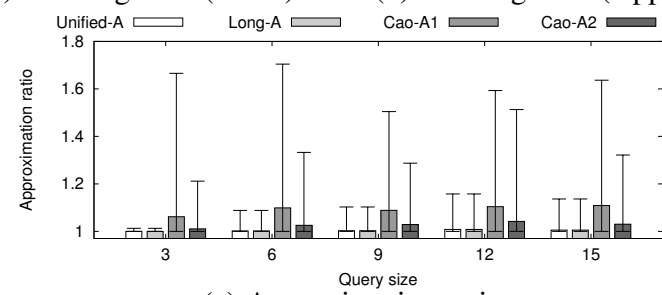

(c) Approximation ratio

Fig. 29: Effect of $|q . \psi|$ on $\operatorname{cost}_{\operatorname{Max} \operatorname{Max} 2}(\mathrm{Web})$

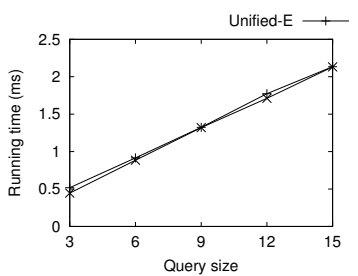

(a) $\mathrm{GN}$

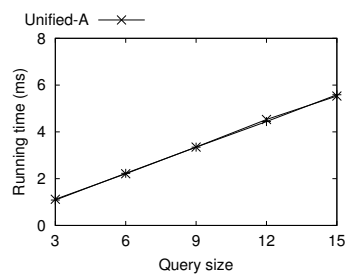

(b) Web
Fig. 30: Effect of $|q . \psi|$ on $\operatorname{cost}_{\text {Max }}$

scalable than Cao-E1, e.g., on a dataset with $6 \mathrm{M}$ objects, Unified$E$ ran for a couple of seconds while Cao-El ran for more than 10 hours. Besides, similar to the case of $\operatorname{cost}_{\text {MinMax }}$, Unified-A runs slightly slower than $\mathrm{Cao}-\mathrm{Al}$, but gives much better approximation ratio, e.g. the median of approximation ratios of Unified-A are 1 on all settings while that of Cao-Al are larger than 1 .

(3) $\operatorname{cost}_{\text {Sum }}$. The results for $\operatorname{cost}_{\text {Sum }}$ are shown in Figure 32. According to Figure 32(a), Unified-E is very scalable when the number of objects is large, e.g., it ran slightly longer than 1 second on a dataset with $10 \mathrm{M}$ objects. Besides, we noticed that Cao-E2 has a very good performance and it even runs as fast as the approximation algorithms. The reason could be as follows. With the number of objects grows, the number of relevant objects 


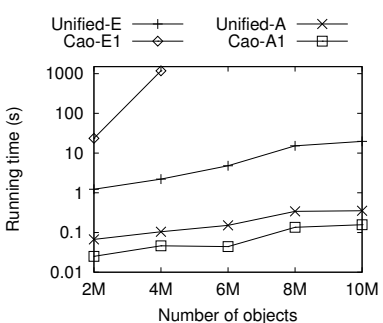

(a) Running time

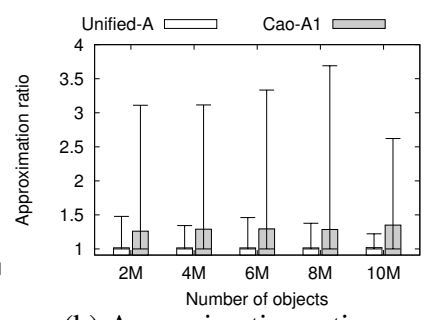

(b) Approximation ratio
Fig. 31: Scalability test on $\operatorname{cost}_{\operatorname{Min} \operatorname{Max} 2}$

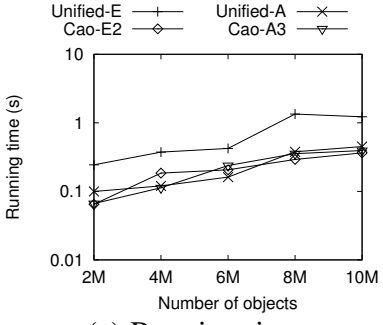

(a) Running time

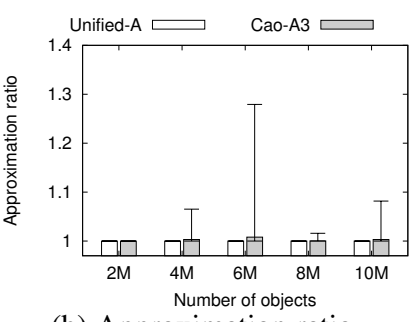

(b) Approximation ratio
Fig. 32: Scalability test on cost $_{\text {Sum }}$

becomes large. Both approximate algorithms have to re-compute the ratio for the remaining nodes in the heap and re-organize the heap after picking each object, whose cost becomes expensive when the number of relevant objects is large. In contrast, Cao-E2 maintains a heap structure though, it does not have to re-examine the nodes after processing a node. Unified- $A$ has similar running times as $\mathrm{CaO}-\mathrm{A} 3$ but gives better approximation ratios than $\mathrm{Cao}-\mathrm{A} 3$ (Figure 32 b)). Specifically, Unified-A can achieve near-to-optimal approximation ratios on all setting while $\mathrm{CaO}-\mathrm{A} 3$ has its largest approximation ratios up to 1.279 .

(4) $\operatorname{cost}_{\text {SumMax }}$. Same as the experiments of varying $|o . \psi|$ for cost $_{\text {SumMax }}$, we used the setting of $|q . \psi|=8$ for the scalability test experiments for cost SumMax $_{\text {particularly. The results for }}$ cost $_{\text {SumMax }}$ are shown in Figure 33. According to Figure 33. a), Unified-E and Cao-E1 have similar running times and Unified-A and $C a o-A 3$ also have similar running times, but Unified-A gives a better approximation ratio than Cao-A3 (Figure 33. b)).

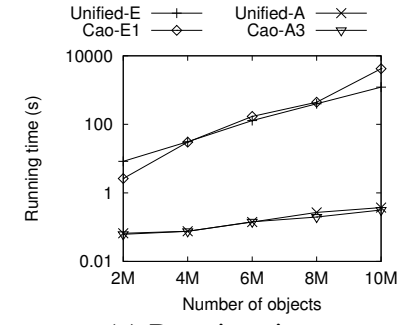

(a) Running time

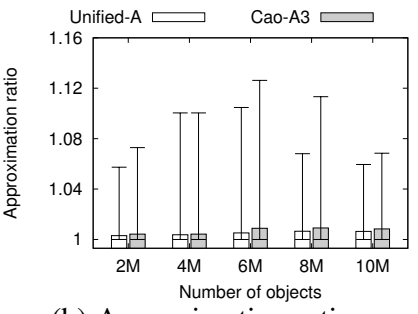

(b) Approximation ratio
Fig. 33: Scalability test on $\operatorname{cost}_{\text {SumMax }}$

(5) $\operatorname{cost}_{\text {MaxMax }}$. The results for cost $_{\text {MaxMax }}$ are shown in Figure 34. According to Figure 34 a), Unified-E runs faster than Long-E but slower than Cao-E1. According to Figure 34 b) and (c), Unified-A runs faster than Long-A and Cao-A2 and slower than Cao-A1, and Unified-A is one of the two algorithms (the other is Long-A which runs slower than Unified-A by about one order of magnitude) which give the best approximation ratios. Specifically, the largest approximation ratios of Unified-A is only 1.134 , which is small, while that of Cao-A1 and Cao-A2 are 2.456 and 1.345 , respectively.

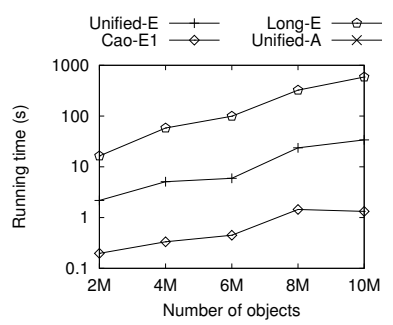

(a) Running time (Exact)

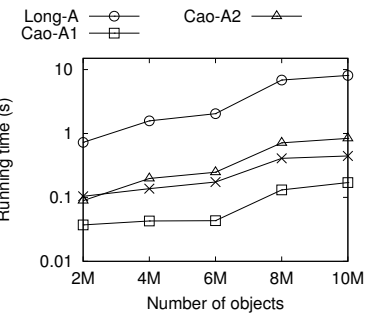

(b) Running time (Appro.)

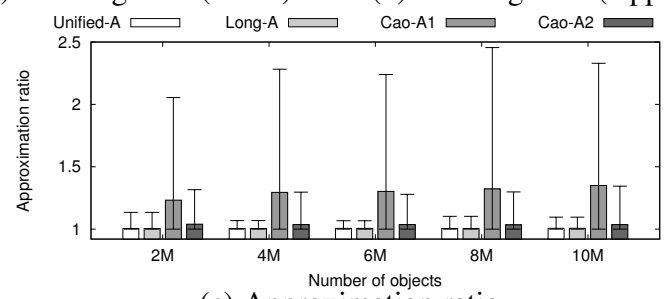

(c) Approximation ratio

Fig. 34: Scalability test on $\operatorname{cost}_{\text {Max Max }}$

(6) $\operatorname{cost}_{M a x M a x 2}$. The results for $\operatorname{cost}_{M a x M a x 2}$ are shown in Figure 35 . According to Figure 35 a), Unified-E runs similarly fast as Long-E, and both of them run faster than Cao-E1. According to Figure 35. b) and (c), Unified-A has similar running times with Cao-A2, both of them run faster than Long-A and slower than Cao-A1, and Unified-A is one of the two algorithms (the other is Long-A which runs slower than Unified-A) which give the best approximation ratios. Specifically, the largest approximation ratios of Unified-A is only 1.109 , which is small, while that of Cao-AI and $C a o-A 2$ are 1.928 and 1.524 , respectively.

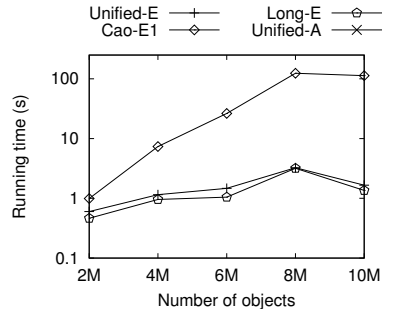

(a) Running time (Exact)

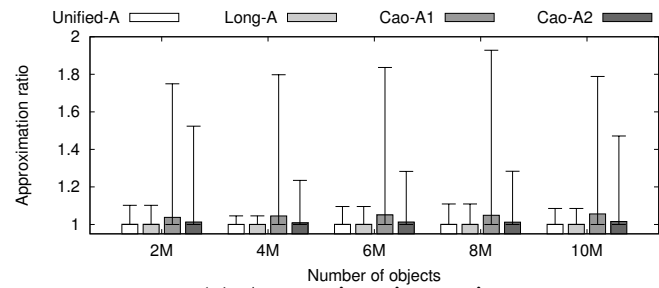

(c) Approximation ratio

Fig. 35: Scalability test on $\operatorname{cost}_{\text {Max Max } 2}$

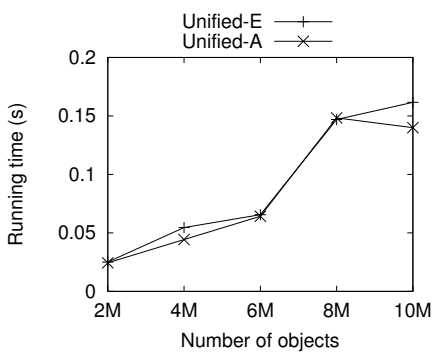

Fig. 36: Scalability test on cost $_{\text {Max }}$
(7) $\operatorname{cost}_{M a x}$. The results for $\operatorname{cost}_{\text {Max }}$ are shown in Figure 36 According to the results, both Unified-E and Unified-A runs very fast, e.g. they ran within 1 second on a dataset with $10 \mathrm{M}$ objects. 